\title{
Coexistence for an Almost Periodic Predator-Prey Model with Intermittent Predation Driven by Discontinuous Prey Dispersal
}

\author{
Yantao Luo, Long Zhang, Zhidong Teng, and Tingting Zheng \\ College of Mathematics and System Science, Xinjiang University, Urumqi 830046, China \\ Correspondence should be addressed to Long Zhang; longzhang_xj@sohu.com
}

Received 26 July 2017; Accepted 23 October 2017; Published 6 December 2017

Academic Editor: Guang Zhang

Copyright ( 2017 Yantao Luo et al. This is an open access article distributed under the Creative Commons Attribution License, which permits unrestricted use, distribution, and reproduction in any medium, provided the original work is properly cited.

\begin{abstract}
An almost periodic predator-prey model with intermittent predation and prey discontinuous dispersal is studied in this paper, which differs from the classical continuous and impulsive dispersal predator-prey models. The intermittent predation behavior of the predator species only happens in the channels between two patches where the discontinuous migration movement of the prey species occurs. Using analytic approaches and comparison theorems of the impulsive differential equations, sufficient criteria on the boundedness, permanence, and coexistence for this system are established. Finally, numerical simulations demonstrate that, for an intermittent predator-prey model, both the intermittent predation and intrinsic growth rates of the prey and predator species can greatly impact the permanence, extinction, and coexistence of the population.
\end{abstract}

\section{Introduction}

In real ecosystems, since the spatial distribution and dynamics of a population are greatly affected by their spatial heterogeneity and population mobility, dispersal becomes one of the dominant themes in mathematical biology. In fact, animal dispersal movements between patches are extremely prevalent in ecological environments; for example, many types of birds and mammals will migrate from cold regions to warm regions in search of a better habitat or a breeding site [1]. Therefore, to take spatial heterogeneity into account, realistic population models should contain the dispersal process. During the past couple of decades, predator-prey models with diffusion in a patchy environment have attracted significant attention from ecologists, biologists, and biomathematicians. Many important works and monographs about the properties of population dynamics in a spatial idiosyncratic environment, for example, permanence, extinction, and global asymptotic stability of positive periodic solutions, have been written (see [2-14]). Teng and Chen [6] considered a nonautonomous predator-prey Lotka-Volterra type dispersal system with periodic coefficients and distributed delays:

$$
\dot{x}_{1}(t)=x_{1}(t)\left[a_{1}(t)-b_{1}(t) x_{1}(t)\right.
$$

$$
\begin{aligned}
& \left.-c(t) \int_{-\infty}^{0} k_{12}(s) y(t+s) d s\right] \\
& +\sum_{j=1}^{n} d_{1 j}(t)\left[x_{j}(t)-x_{1}(t)\right], \\
& \dot{x}_{i}(t)=x_{i}(t)\left[a_{i}(t)-b_{i}(t) x_{i}(t)\right]+\sum_{j=1}^{n} d_{i j}(t)\left[x_{j}(t)\right. \\
& \left.-x_{i}(t)\right], \quad i=2,3, \ldots, n, \\
& \dot{y}(t)=y(t)\left[-e(t)+f(t) \int_{-\infty}^{0} k_{21}(s) x_{1}(t+s) d s\right. \\
& \left.-g(t) \int_{-\infty}^{0} k_{21}(s) x_{1}(t+s) d s\right],
\end{aligned}
$$

where $y$ is the population density of the predator species confined to the 1st patch. Criteria for the permanence, extinction, and existence of positive periodic solutions for system (1) were established. In this model, the prey dispersal behavior occurs at every point in time and simultaneously 
between any patches; that is, it is a continuous bidirectional dispersal.

However in practice, it is often the case that diffusion occurs in the form of regular pulses. For example, when winter comes, birds will migrate between patches to seek a better habitat, whereas they do not diffuse in other seasons, and the dispersion of foliage seeds occurs at a fixed period of time every year. For another example, in the Pacific Northwest, Larimichthys polyactis cross over deep water during the winter and migrate to the coast during the spring; then, 3-6 months after spawning, they scatter offshore and return to the depths of the sea during late autumn [15]. All these types of migratory behaviors are appropriately assumed to be in the form of pulses in the modeling process. Thus, impulsive diffusion provides a more natural description. Currently, theories of impulsive differential equations [16] have been introduced into population dynamics. A large number of models have been described by impulsive diffusion (see [14, 17-24]) during the past couple of decades.

Shao [23] considered the following predator-prey models with impulsive prey diffusion between two patches:

$$
\begin{aligned}
& \dot{x}_{1}(t)=x_{1}(t)\left(r_{1}-a_{1} x_{1}(t)-b_{1} y(t)\right), \\
& \dot{x}_{2}(t)=x_{2}(t)\left(r_{2}-a_{2} x_{2}(t)\right), \\
& \dot{y}(t)=y(t)\left(-r_{3}+a_{3} x_{1}\left(t-\tau_{1}\right)-b_{2} y\left(t-\tau_{2}\right)\right),
\end{aligned}
$$
$t \neq n \tau$,

$$
\begin{aligned}
& \Delta x_{1}(t)=d_{1}\left(x_{2}(t)-x_{1}(t)\right), \\
& \Delta x_{2}(t)=d_{2}\left(x_{1}(t)-x_{2}(t)\right), \\
& \Delta y(t)=0,
\end{aligned}
$$$$
t=n \tau
$$

where the pulse diffusion of the species $x$ occurs in every period $T$ (a positive constant) and $d_{i}$ is the dispersal rate in the $i$ th patch satisfying $0<d_{i}<1$ for $i=1,2$. The system evolves from its initial state without being further affected by diffusion until the next pulse appears. $\Delta x_{i}(n T)=x_{i}\left(n T^{+}\right)-x_{i}(n T)$, where $x_{i}\left(n T^{+}\right)$represents the density of the population of the prey species $x$ in the $i$ th patch immediately after the $n$th diffusion pulse at time $t=n T ; x_{i}(n T)$ represents the density of the population of the prey species in the $i$ th patch before the $n$th diffusion pulse at time $t=n T, n=0,1, \ldots$. Criteria for the global attractivity and permanence of system (2) were obtained.

Furthermore, migration movements of the population will be influenced by many uncertain factors (such as the landscape and weather). Therefore, the dispersal movement of migratory species will have to be suspended when the environment becomes unavailable. In other words, patches would permit normal movement patterns between patches to occur only during certain time intervals instead of all the time. For instance, in the Canary Islands of Spain, Anas platyrhynchos undergo a spring migration from early March to the end of March and a fall migration from late September to the end of October, departing as late as early November, during which they are extensively killed by humans, and other carnivorous animals can prolong the journey [25]. As another example, the wild goose will fly to the south when winter comes; in this process, they will stop to rest in some places and at certain time periods. In other words, their diffusion behavior is neither continuous at all times nor impulsive at a fixed time, but it is intermittent within some time intervals. Therefore, it is more reasonable to model this kind of population dynamics with intermittent dispersals. Zhang et al. [26] considered the following nonautonomous almost periodic single species model with intermittent dispersals and dispersal delays between two patches:

$$
\begin{aligned}
& \dot{x}_{1}(t)=x_{1}(t)\left[a_{1}(t)-b_{1}(t) x_{1}(t)\right], \\
& \dot{x}_{2}(t)=x_{2}(t)\left[a_{2}(t)-b_{2}(t) x_{2}(t)\right] \text {, } \\
& t \in\left[\tau_{2 k}, \tau_{2 k+1}\right), \\
& x_{1}\left(\tau_{2 k+1}\right)=d_{1} x_{1}\left(\tau_{2 k+1}^{-}\right) \text {, } \\
& x_{2}\left(\tau_{2 k+1}\right)=d_{2} x_{2}\left(\tau_{2 k+1}^{-}\right) \text {, } \\
& \dot{x}_{1}(t)=x_{1}(t)\left[\tilde{a}_{1}(t)-\widetilde{b}_{1}(t) x_{1}(t)\right] \\
& +D_{12}(t)\left(x_{2}\left(t-\tau_{1}\right)-x_{1}(t)\right) \text {, } \\
& \dot{x}_{2}(t)=x_{2}(t)\left[\tilde{a}_{2}(t)-\tilde{b}_{2}(t) x_{2}(t)\right] \\
& +D_{21}(t)\left(x_{1}\left(t-\tau_{2}\right)-x_{2}(t)\right) \text {, } \\
& t \in\left[\tau_{2 k+1}, \tau_{2 k+2}\right), \\
& t=\tau_{2 k+1} \text {, } \\
& x_{1}\left(\tau_{2 k+2}\right)=D_{1} x_{1}\left(\tau_{2 k+2}^{-}\right), \\
& x_{2}\left(\tau_{2 k+2}\right)=D_{2} x_{2}\left(\tau_{2 k+2}^{-}\right) \text {, } \\
& t=\tau_{2 k+2},
\end{aligned}
$$

where all the parameters are almost periodic and the dispersal movement happens only in the time interval $t \in\left[\tau_{2 k+1}, \tau_{2 k+2}\right)$ but not in $t \in\left[\tau_{2 k}, \tau_{2 k+1}\right)$. Here, $k \in N$. Criteria for the existence, uniqueness, and global attractivity of positive almost periodic solution for system (3) were established.

Moreover, in a real ecological system, there always exist natural enemies during the migratory process between patches. For example, annually, at the end of July, with the arrival of the dry season, millions of wildebeests, zebras, and other herbivorous wildlife form a migratory army, migrating from the Serengeti National Park, Tanzania, Africa, to Kenya's Masai Mara National Nature Reserve to find enough water and food. Along the way, they will be preyed upon by lions, leopards, and so on. Additionally, crocodiles and hippopotamus will wait and ambush the migration species in the Mara River. As the seasons alternate, i.e., when the rainy season comes, the migration movement starts again, and these species will return to the Serengeti National Park, and vice versa [27]. Obviously, the predation behavior for the above situation is intermittent and only happens in the channels where the dispersals of migratory species occur. 
Motivated by the above consideration, in this paper, we introduce an almost periodic predator-prey model with intermittent predation and discontinuous prey dispersal between two patches:

$$
\begin{aligned}
& \dot{x}_{1}(t)=x_{1}(t)\left[a_{1}(t)-b_{1}(t) x_{1}(t)\right], \\
& \dot{x}_{2}(t)=x_{2}(t)\left[a_{2}(t)-b_{2}(t) x_{2}(t)\right] \text {, } \\
& \dot{y}(t)=y(t)\left[a_{3}(t)-b_{3}(t) y(t)\right], \\
& t \in\left[\tau_{2 k}, \tau_{2 k+1}\right) \\
& x_{1}\left(\tau_{2 k+1}\right)=d_{1} x_{1}\left(\tau_{2 k+1}^{-}\right) \text {, } \\
& x_{2}\left(\tau_{2 k+1}\right)=d_{2} x_{2}\left(\tau_{2 k+1}^{-}\right) \text {, } \\
& y\left(\tau_{2 k+1}\right)=y\left(\tau_{2 k+1}^{-}\right), \\
& t=\tau_{2 k+1}, \\
& \dot{x}_{1}(t)=x_{1}(t)\left[\tilde{a}_{1}(t)-\tilde{b}_{1}(t) x_{1}(t)-c_{1}(t) y(t)\right] \\
& +D_{12}(t)\left(x_{2}\left(t-\tau_{1}\right)-x_{1}(t)\right) \\
& \dot{x}_{2}(t)=x_{2}(t)\left[\tilde{a}_{2}(t)-\widetilde{b}_{2}(t) x_{2}(t)-c_{2}(t) y(t)\right] \\
& +D_{21}(t)\left(x_{1}\left(t-\tau_{2}\right)-x_{2}(t)\right) \\
& \dot{y}(t)=y(t) \\
& \cdot\left[\tilde{a}_{3}(t)-\widetilde{b}_{3}(t) y(t)+\widetilde{c}_{1}(t) x_{1}(t)+\widetilde{c}_{2}(t) x_{2}(t)\right], \\
& t \in\left[\tau_{2 k+1}, \tau_{2 k+2}\right), \\
& x_{1}\left(\tau_{2 k+2}\right)=D_{1} x_{1}\left(\tau_{2 k+2}^{-}\right) \text {, } \\
& x_{2}\left(\tau_{2 k+2}\right)=D_{2} x_{2}\left(\tau_{2 k+2}^{-}\right) \text {, } \\
& y\left(\tau_{2 k+2}\right)=y\left(\tau_{2 k+2}^{-}\right),
\end{aligned}
$$

where $x_{i}(t)$ denotes the prey population density in the $i$ th patch $(i=1,2)$ and $y(t)$ represents the predator population density in channels between two patches. When $t \in$ $\left[\tau_{2 k}, \tau_{2 k+1}\right)$ with $k \in N$, the prey species $x_{i}$ inhabits the $i$ th patch and does not disperse. At the same time, the predator species $y$ inhabits the channels between two patches with other food sources. When $t \rightarrow \tau_{2 k+1}$, the intrinsic discipline of the species $x$ in each patch changes. The channels between the two patches will open, and the species $x$ disperses bidirectionally from one patch to another; this dispersal movement will continue for the time interval $t \in$ $\left[\tau_{2 k+1}, \tau_{2 k+2}\right)$. Meanwhile, the predator species $y$ preys on the species $x$ in the channels. When $t \rightarrow \tau_{2 k+2}$, the gate of the channels will close, the species $x_{i}$ will stop dispersing and inhabit patch $i$. At the same time, the predator species $y$ will also stop preying on species $x$. Obviously, the predation behavior only happens in the time interval $t \in\left[\tau_{2 k+1}, \tau_{2 k+2}\right)$; that is, it is intermittent. Here, $a_{i}(t), \widetilde{a}_{i}(t)(i=1,2)$ represent the intrinsic growth rates of the species $x$ in the $i$ th patch over the time intervals $\left[\tau_{2 k}, \tau_{2 k+1}\right)$ and $\left[\tau_{2 k+1}, \tau_{2 k+2}\right)$, respectively; $a_{3}(t), \tilde{a}_{3}(t)$ denote the intrinsic growth rates of the species $y$ over the time intervals $\left[\tau_{2 k}, \tau_{2 k+1}\right)$ and $\left[\tau_{2 k+1}, \tau_{2 k+2}\right)$, respectively; $b_{i}(t), \widetilde{b}_{i}(t)(i=1,2)$ represent the intercompetition rates of the species $x$ in the $i$ th patch over the time intervals $\left[\tau_{2 k}, \tau_{2 k+1}\right)$ and $\left[\tau_{2 k+1}, \tau_{2 k+2}\right)$, respectively; $b_{3}(t), \widetilde{b}_{3}(t)$ denote the intercompetition rates of the species $y$ over the time intervals $\left[\tau_{2 k}, \tau_{2 k+1}\right)$ and $\left[\tau_{2 k+1}, \tau_{2 k+2}\right)$, respectively; $d_{i}$ represents the survival rates of switching from stage 1 (without dispersal) to stage 2 (dispersal movement); $D_{i}$ denotes the survival rates of switching from stage 2 to stage $1 ; D_{i j}(t)$ is the dispersal rates from the $i$ th patch to the $j$ th patch during the time interval $\left[\tau_{2 k+1}, \tau_{2 k+2}\right)$; and $\tau_{i}$ is the time for the population to disperse from patch $j$ to $i(i \neq j, i, j=1,2)$. During the whole process, the predator species $y$ never disperses.

In allusion to system (4) above, our main purpose in this paper is to establish a series of criteria on the ultimate boundedness, permanence, and coexistence of the two populations for system (4). The methods used in this paper are motivated by the works on the permanence and extinction for periodic predator-prey systems in patchy environments given by Teng and Chen in [6] and the works on the survival analysis for a periodic predator-prey model given by Zhang et al. in [22].

This paper is organized as follows. In Section 2, some definitions, assumptions, and useful lemmas are introduced. In Section 3, we state and prove the main results. Finally, special examples and numerical simulations are illustrated to demonstrate our theoretical results in Section 4.

\section{Preliminaries}

Let $R$ and $R^{2}$ denote the set of real numbers and the 2dimensional Euclidean linear space, respectively, and $\tau_{k}$ be a time sequence, satisfying $\tau_{k}<\tau_{k+1}$, with $\tau_{k} \rightarrow \infty$ as $k \rightarrow \infty$. Here, $k \in N$. Define $\widehat{\tau}=\max \left\{\tau_{1}, \tau_{2}\right\}, P C\left([t-\widehat{\tau}, t], R^{2}\right)=$ $\left\{\psi:\left([t-\widehat{\tau}, t] \rightarrow R^{2}\right) \mid \varphi=\left(\varphi_{1}, \varphi_{2}\right)\right.$ is continuous everywhere except at $t=\tau_{k} \in[t-\widehat{\tau}, t]$, and $\varphi\left(\tau_{k}^{+}\right), \varphi\left(\tau_{k}^{-}\right)$exist with $\varphi\left(\tau_{k}^{+}\right)=$ $\left.\varphi\left(\tau_{k}\right)\right\}$. Define BPC $=\left\{\varphi \in P C\left((-\infty, 0], R^{2}\right]: \varphi\right.$ is bounded $\}$; the norm of $\varphi$ is defined by $\|\varphi\|=\sup _{\theta \in[-\widehat{\tau}, 0]}|\varphi(\theta)|$. Let $B P C_{+}=\left\{\varphi=\left(\varphi_{1}, \varphi_{2}\right) \in B P C: \varphi_{i}(\theta) \geq 0\right.$ for all $\theta \in[-\infty, 0]$ and $\varphi_{i}\left(0_{+}\right)>0$ for $\left.i=1,2\right\}$.

In this paper, we assume that all solutions of system (4) satisfy the following initial conditions:

$$
\begin{aligned}
x_{i}(s, \phi) & =\phi_{i}(s), \\
s & \in[-\widehat{\tau}, 0], \\
x_{i}\left(0^{+}, \phi\right) & =\phi_{i}\left(0^{+}\right), \\
s & \in[-\widehat{\tau}, 0],
\end{aligned}
$$

where $\phi=\left(\phi_{1}, \phi_{2}\right) \in B P C_{+}$. It is not hard to prove that the functional of right of system (4) is continuous and satisfies the local Lipschitz condition with respect to $\phi$ in the space $R \times$ $B P C$. Therefore, by the fundamental theory of the impulsive functional differential equations with finite delays $[16,28$, 29], system (4) has a unique solution $\left(x_{1}(t, \phi), x_{2}(t, \phi), y(t)\right)$ satisfying the initial conditions (5). Obviously, the solution 
$\left(x_{1}(t, \phi), x_{2}(t, \phi), y(t)\right)$ is positive in its maximal interval of the existence.

Before going into details, we first draw some very useful definitions and lemmas.

Definition 1 (see [30]). The set of sequences $\left\{\tau_{k}{ }^{i} \mid \tau_{k}{ }^{i}=\tau_{k+1}-\right.$ $\left.\tau_{k}, k, i \in N, \tau_{k} \in B\right\}$ is said to be uniformly almost periodic if, for arbitrary $\varepsilon>0$, there exists a relatively dense set in $R$ of $\varepsilon$-almost periodic common for all of the sequences; here $B=\left\{\tau_{k} \mid \tau_{k} \in R, \tau_{k}<\tau_{k+1}, k \in N, \lim _{k \rightarrow \pm \infty} \tau_{k}= \pm \infty\right\}$.

Definition 2 (see [30]). Assume that the following conditions hold:

(1) The set of sequences $\left\{\tau_{k}^{i}\right\}$ is almost periodic, $k, i \in N$.

(2) For any $\varepsilon>0$ there exists a real number $\delta>0$ such that if the points $t^{\prime}, t^{\prime \prime}$ belong to the same interval of continuity of $\varphi(t)$ and satisfy the inequality $\left|t^{\prime}-t^{\prime \prime}\right|<$ $\delta$, then $\left|\varphi\left(t^{\prime}\right)-\varphi\left(t^{\prime \prime}\right)\right|<\varepsilon$.

(3) For any $\varepsilon>0$ there exists a relatively dense set $C$ such that if $\sigma \in C$, then $|\varphi(t+\sigma)-\varphi(t)|<\varepsilon$ for all $t \in$ $R$ satisfying the condition $\left|t-\sigma_{k}\right|>\varepsilon, k \in N$. The elements of $C$ are called $\epsilon$-almost periods.

We claim that function $\varphi \in P C\left([t-\widehat{\tau}, t], R^{2}\right)$ is almost periodic, and we denote $\varphi \in A P C$.

Definition 3 (see [31]). System (4) is said to be permanent if there exist positive constants $m$ and $M$, such that for any positive solutions $x(t)=\left(x_{1}(t), x_{2}(t), y(t)\right)$ of system (4) with initial value that satisfy condition (5)

$$
\begin{aligned}
& m \leq \liminf _{t \rightarrow \infty} x_{i}(t) \leq \limsup _{t \rightarrow \infty} x_{i}(t) \leq M, \quad i=1,2, \\
& m \leq \liminf _{t \rightarrow \infty} y(t) \leq \limsup _{t \rightarrow \infty} y(t) \leq M .
\end{aligned}
$$

Definition 4 (see [24]). Let $V: R \times R^{2} \rightarrow R^{2}$, then $V$ is said to belong to class $L$ if

(i) $V$ is continuous in $\left(\tau_{k}, \tau_{k+1}\right) \times R^{2}$ for each $x \in R^{2}, k \in$ $N$,

$$
\lim _{(t, y) \rightarrow\left(\tau_{k+1}^{-}, x\right)} V(t, y)=V\left(\tau_{k+1}, x\right) .
$$

(ii) $V$ is locally Lipschitzian in $x$.

In this paper, there are some notations and assumptions that shall be used:

$\left(N_{1}\right)$ If $f(t), t \in R$, is an almost periodic function, we define

$$
\begin{aligned}
A(f) & =\lim _{t \rightarrow \infty} \frac{1}{T} \int_{t}^{t+T} f(s) d s, \\
f^{u} & =\sup _{t \in R} f(t), \\
f^{l} & =\inf _{t \in R} f(t),
\end{aligned}
$$

where $T$ is a positive constant.
$\left(N_{2}\right)$ We let $\lambda_{i}(t)=t-\tau_{i}, \lambda_{i}^{-1}(t)$ be an inverse function of the function $\lambda_{i}(t)(i=1,2)$.

$\left(H_{1}\right)$ Functions $a_{i}(t), \widetilde{a}_{i}(t), b_{i}(t), \widetilde{b}_{i}(t)$, and $D_{i j}(t)$ are $\tau$ almost periodic and bounded continuous functions for all $t \in R$ and $b_{i}^{l} \geq 0, \tilde{b}_{i}^{l} \geq 0,0<d_{i}, D_{i} \leq 1(i=$ $1,2,3, j=1,2)$.

$\left(H_{2}\right) A\left(a_{i}(t)\right)>0, A\left(\widetilde{a}_{i}(t)\right)>0, A\left(b_{i}(t)\right)>0, A\left(\widetilde{b}_{i}(t)\right)>$ $0(i=1,2)$.

$\left(H_{3}\right)$ There exists a constant $\tau>0$, such that, for any $t \geq 0$,

$$
\begin{aligned}
\int_{t}^{t+\tau} & {\left[\frac{(-1)^{k}+1}{2} a_{i}(s)+\frac{(-1)^{k+1}+1}{2} \widetilde{a}_{i}(s)\right] d s } \\
& +\sum_{t<\tau_{k+1} \leq(t+\tau)} \ln h_{i k}>0 \quad(i=1,2, k \in N) .
\end{aligned}
$$

$\left(H_{4}\right)$ There exists a constant $\xi_{i}>0(i=1,2)$, such that, for any $t \geq 0$,

$$
\left|\sum_{t<\tau_{k+1} \leq(t+s)} \ln h_{i k}\right| \leq \xi_{i}, \quad s \in[0, \tau] .
$$

$\left(H_{5}\right) A\left(a_{i k}(t)-d_{i j k}(t)\right)>0(i, j=1,2, k \in N)$.

$\left(H_{6}\right)$ There exist constants $\theta>0, e_{i}>0(i=1,2)$, such that $\lim _{t \rightarrow \infty} \inf B_{i}(t)=\lim _{t \rightarrow \infty}\left[e_{i}\left(b_{i k}(t)\right)-\right.$ $\left.d_{i j k}\left(\lambda_{j}{ }^{-1}(t)\right) / \widetilde{m}\right] \geq \theta$, where $\widetilde{m}=\max \{1,[m]\}$ and $[m]$ is the integer part of $m(k \in N)$.

$\left(H_{7}\right)$ The set of sequences $\left\{\tau_{k}^{i}\right\}, k \in N$, is uniformly almost periodic, and $\tau_{k+2}=\tau_{k}+\tau, \inf _{k \in N}\left|\tau_{k+1}-\tau_{k}\right|>0$, where $\tau$ belongs to the relatively dense set $T$ ( $i=$ $1,2,3)$.

$\left(H_{8}\right)$ There exists a constant $\tilde{\tau}>0$, such that, for any $t \geq 0$,

$$
\int_{t}^{t+\tilde{\tau}}\left[\frac{(-1)^{k}+1}{2} a_{3}(s)+\frac{(-1)^{k+1}+1}{2} \widetilde{a}_{3}(s)\right] d s>0 .
$$

Now, we give some useful lemmas which will be used in the proofs of the main results.

If there is no predator $y$ in system (4), we have the following predator-free system:

$$
\begin{array}{rl}
\dot{x}_{i}(t)= & x_{i}(t)\left[a_{i k}(t)-b_{i k}(t) x_{i}(t)\right] \\
& +d_{i j k}(t)\left[x_{j}\left(t-\tau_{i}\right)-x_{i}(t)\right], \\
t & t \in\left[\tau_{k}, \tau_{k+1}\right),
\end{array}
$$

$x_{i}\left(\tau_{k+1}\right)=h_{i k} x_{i}\left(\tau_{k+1}^{-}\right), \quad t=\tau_{k+1}$, 
where

$$
\begin{aligned}
a_{i k}(t) & =\frac{(-1)^{k}+1}{2} a_{i}(t)+\frac{(-1)^{k+1}+1}{2} \widetilde{a}_{i}(t), \\
b_{i k}(t) & =\frac{(-1)^{k}+1}{2} b_{i}(t)+\frac{(-1)^{k+1}+1}{2} \widetilde{b}_{i}(t), \\
d_{i j k}(t) & =\frac{(-1)^{k+1}+1}{2} D_{i j}(t), \\
h_{i k} & =\frac{(-1)^{k}+1}{2} d_{i}+\frac{(-1)^{k+1}+1}{2} D_{i}, \\
& \quad i \neq j, i, j=1,2 .
\end{aligned}
$$

For system (12), we have the following result.

Lemma 5 (see [26] Theorem 3.4). Suppose that assumptions $\left(H_{1}\right)-\left(H_{7}\right)$ hold; then system (12) has a unique globally attractive positive $\tau$-almost periodic solution $x^{*}(t)=\left(x_{1}^{*}(t), x_{2}^{*}(t)\right)$.

If there is no prey $x$ in system (4), we have the following prey-free system:

$$
\begin{aligned}
\dot{y}(t) & =y(t)\left[a_{3 k}(t)-b_{3 k}(t) y(t)\right], \\
t \in\left[\tau_{k}, \tau_{k+1}\right), & \\
y\left(\tau_{k+1}\right) & =y\left(\tau_{k+1}^{-}\right), \quad t=\tau_{k+1},
\end{aligned}
$$

where

$$
\begin{aligned}
& a_{3 k}(t)=\frac{(-1)^{k}+1}{2} a_{3}(t)+\frac{(-1)^{k+1}+1}{2} \widetilde{a}_{3}(t), \\
& b_{3 k}(t)=\frac{(-1)^{k}+1}{2} b_{3}(t)+\frac{(-1)^{k+1}+1}{2} \widetilde{b}_{3}(t) .
\end{aligned}
$$

For system (14), we have the following result.

Lemma 6 (see [26]). Suppose assumptions $\left(H_{1}\right)-\left(H_{2}\right)$ and $\left(H_{7}\right)-\left(H_{8}\right)$ hold; then system (14) has a unique globally attractive positive $\tau$-almost periodic solution $y^{*}(t) \in$ APC.

$$
\begin{aligned}
& \text { For }(t, x) \in\left(\tau_{k}, \tau_{k+1}\right) \times R^{2} \text {, we define } \\
& \begin{aligned}
D^{+} V(t, x) \\
\quad=\limsup _{h \rightarrow 0^{+}} \frac{1}{h}[V(t+h, x+h f(t, x))-V(t, x)],
\end{aligned}
\end{aligned}
$$

where $f=\left(f_{1}, f_{2}\right)$ is the right-hand side of system (12). We give the following vector comparison results of the impulsive differential equations.

Lemma 7 (see [1]). Let $V: R_{+} \times R_{+}^{2}$ and $V \in L$. Assume that

$$
\begin{array}{ll}
D^{+} V(t, x) \leq g(t, V(t, x)) & t \in\left[\tau_{k}, \tau_{k+1}\right), \\
V(t, x(t)) \leq \psi_{k}(V(t, x)), & t=\tau_{k+1},
\end{array}
$$

where $g(t, u): R \times R^{2} \rightarrow R^{2}$ is continuous in $\left(\tau_{k}, \tau_{k+1}\right) \times R^{2}$ and quasi-monotone nondecreasing in $u$, for $v \in R^{2}, \lim _{(t, u) \rightarrow\left(\tau_{k+1}^{-}\right)} g(t, u)=g\left(\tau_{k+1}^{-}, v\right)$ exists, and $\psi_{k}: R^{2} \rightarrow$ $R^{2}$ is nondecreasing. Let $r(t)$ be the maximal solution of the following vector impulsive differential system:

$$
\begin{aligned}
\dot{u} & =g(t, V(t, x)) \quad t \in\left[\tau_{k}, \tau_{k+1}\right), \\
u(t) & =\psi_{k}\left(u\left(t^{-}\right)\right), \\
u\left(t_{0}\right) & =u\left(t_{0}^{-}\right)=u_{0} \quad \\
& t=\tau_{k+1},
\end{aligned}
$$

existing on $\left[t_{0}, \infty\right)$. Then $V\left(t_{0}, x_{0}\right) \leq u_{0}$ implies that

$$
V(t, x(t)) \leq r(t), \quad t \geq t_{0},
$$

where $x(t)=x\left(t, t_{0}, x_{0}\right)$ is any solution of system (12) existing on $\left[t_{0}, \infty\right)$. Here, we state that function $g(t, u)$ is quasimonotone nondecreasing in $u$; if $u, v \in R^{2}, u \leq v$, and $x_{i}=y_{i}$ for some $1 \leq i \leq 2$, then $g_{i}(t, x) \leq g_{i}(t, y)$.

\section{Main Results}

First, in terms of the ultimate boundedness for system (4), we obtain the following result.

Theorem 8. Suppose that assumptions $\left(H_{1}\right)-\left(H_{9}\right)$ hold. Then there is a constant $M>0$ such that

$$
\begin{gathered}
\lim _{t \rightarrow \infty} \sup x_{i}(t, \phi) \leq M \quad(i=1,2), \\
\lim _{t \rightarrow \infty} \sup y(t) \leq M
\end{gathered}
$$

for any positive solutions $\left(x_{1}(t, \phi), x_{2}(t, \phi), y(t, \phi)\right)$ of system (4).

Proof. Let $\left(x_{1}(t, \phi), x_{2}(t, \phi), y(t)\right)$ be any positive solution of system (4) satisfying the initial condition (5). From system (4), we have

$$
\begin{aligned}
\dot{x}_{i}(t) \leq & x_{i}(t)\left[a_{i k}(t)-b_{i k}(t) x_{i}(t)\right] \\
& +d_{i j k}(t)\left[x_{j}\left(t-\tau_{i}\right)-x_{i}(t)\right], \\
\quad t \in\left[\tau_{k}, \tau_{k+1}\right), & \\
x_{i}\left(\tau_{k+1}\right)= & h_{i k} x_{i}\left(\tau_{k+1}^{-}\right), \quad t=\tau_{k+1},
\end{aligned}
$$

for all $t \geq 0, i, j=1,2(i \neq j)$. Then by the Lemmas 5 and 7 , we obtain

$$
x_{i}(t) \leq \bar{x}_{i}(t), \quad \forall t \geq 0, \quad i=1,2,
$$

where $\left(\bar{x}_{1}(t), \bar{x}_{2}(t)\right)$ is the solution of system (12) with the initial condition (5). Under assumptions $\left(H_{1}\right)-\left(H_{7}\right)$, from Lemma 5 we obtain $\bar{x}_{i}(t) \rightarrow x^{*}(t)(i=1,2, t \rightarrow \infty)$, where $x^{*}(t)=\left(x_{1}^{*}(t), x_{2}^{*}(t)\right)$ is the globally asymptotically stable positive $\tau$-almost periodic solution of system (12). Hence, $\bar{x}_{i}(t)(i=1,2)$ is bounded on $R_{+}$. Next, we choose a constant $M_{1}=\sup _{t \in R}\left|x^{*}(t)\right|$, where $\left|x^{*}(t)\right|=\sum_{i=1}^{2} x_{i}^{*}(t)$. Since $\bar{x}_{i}(t) \rightarrow$ 
$x^{*}(t)(i=1,2, t \rightarrow \infty)$, there is a pair of $T_{1}>0$ and $\rho>0$ such that

$$
\bar{x}_{i}(t) \leq x_{i}^{*}(t)+\rho \triangleq M_{1} \quad \forall t \geq T_{1}, \quad i=1,2 .
$$

Hence, by (22) we have

$$
x_{i}(t) \leq M_{1} \quad \forall t \geq T_{1}, i=1,2 .
$$

Consequently,

$$
\lim _{t \rightarrow \infty} \sup x_{i}(t, \phi) \leq M_{1}, \quad i=1,2 .
$$

Further, we prove that there is a constant $M_{2}>0$ such that

$$
\lim _{t \rightarrow \infty} \sup y(t) \leq M_{2} \text {. }
$$

From systems (4) and (24) we obtain

$$
\begin{aligned}
& \dot{y}(t) \leq y(t) \\
& \cdot\left[a_{3 k}(t)+\widetilde{c}_{1 k}(t) M_{1}+\widetilde{c}_{2 k}(t) M_{2}-b_{3 k}(t) y(t)\right], \\
& t \in\left[\tau_{k}, \tau_{k+1}\right), \\
& y\left(\tau_{k+1}\right)=y\left(\tau_{k+1}^{-}\right), \quad t=\tau_{k+1},
\end{aligned}
$$

\section{$k \in N$.}

From the comparison theorem of the impulsive differential equations $[16,28,29]$, we have $y(t) \leq z(t)$ for all $t \geq T_{1}$, where $z(t)$ is the solution of the following auxiliary equation:

$$
\begin{gathered}
\dot{z}(t)=z(t) \\
\cdot\left[a_{3 k}(t)+\widetilde{c}_{1 k}(t) M_{1}+\widetilde{c}_{2 k}(t) M_{2}-b_{3 k}(t) z(t)\right], \\
t \in\left[\tau_{k}, \tau_{k+1}\right), \\
z\left(\tau_{k+1}\right)=z\left(\tau_{k+1}^{-}\right), \quad t=\tau_{k+1},
\end{gathered}
$$

with initial value $z\left(T_{1}\right)=y\left(T_{1}\right)$. According to assumptions $\left(H_{1}\right)-\left(H_{2}\right)$ and $\left(H_{7}\right)-\left(H_{8}\right)$, we also have

$$
\begin{aligned}
& \int_{t}^{t+\tau}\left\{\frac{(-1)^{k}+1}{2} a_{3}(s)+\frac{(-1)^{k+1}+1}{2}\left[\widetilde{a}_{3}(s)\right.\right. \\
& \left.\left.+\widetilde{c}_{1 k}(t) M_{1}+\widetilde{c}_{2 k}(t) M_{1}\right]\right\} d s>0 .
\end{aligned}
$$

Hence from Lemma 6, system (29) has a unique globally attractive positive $\tau$-almost periodic solution $z^{*}(t)$. For any constant $\lambda>0$, there is a $T_{2} \geq T_{1}$ such that $z(t)<z^{*}(t)+\lambda$ for all $t \geq T_{2}$. Therefore, we have

$$
y(t)<z^{*}(t)+\lambda \leq \frac{1}{2} \max _{t \in[0, \tau]} z^{*}(t)+\lambda \triangleq M_{2},
$$

for all $t \geq T_{2}$. Consequently,

$$
\lim _{t \rightarrow \infty} \sup y(t) \leq M_{2} \text {. }
$$

Therefore, (26) holds. Choose a constant $M=\max \left\{M_{1}, M_{2}\right\}$; then we can see

$$
\begin{gathered}
\lim _{t \rightarrow \infty} \sup x_{i}(t, \phi) \leq M \quad(i=1,2), \\
\lim _{t \rightarrow \infty} \sup y(t) \leq M
\end{gathered}
$$

this completes the proof of Theorem 8 .

Next, on the permanence of system (4), we have the following results.

Theorem 9. Suppose that assumptions $\left(H_{1}\right)-\left(H_{2}\right)$ and $\left(H_{7}\right)$ $\left(H_{8}\right)$ hold. Then the predator species $y$ of system (4) is permanent.

Proof. Let $\left(x_{1}(t, \phi), x_{2}(t, \phi), y(t)\right)$ be any positive solutions of system (4) satisfying the initial condition (5). By system (4), we easily obtain

$$
\begin{array}{cl}
\dot{y}(t) \geq y(t)\left[a_{3 k}(t)-b_{3 k}(t) y(t)\right], & \\
t \in\left[\tau_{k}, \tau_{k+1}\right), \\
y\left(\tau_{k+1}\right)=y\left(\tau_{k+1}^{-}\right), \quad t=\tau_{k+1} .
\end{array}
$$

Using comparison theorem of the impulsive differential equations [16, 28, 29] and Lemma 6 , we can easily obtain $y(t) \geq \bar{y}(t)$, for all $t \in R$, where $\bar{y}(t)$ is the solution of system (14) with condition (5). Under the assumptions $\left(H_{1}\right)-\left(H_{2}\right)$ and $\left(H_{7}\right)-\left(H_{8}\right)$, by Lemma 6 , we can obtain $\bar{y}(t) \rightarrow y^{*}(t)$ as $t \rightarrow \infty$, where $y^{*}(t)$ is the globally asymptotically stable positive $\tau$-almost periodic solution of system (14). Hence, we can easily obtain that, for $\varepsilon_{0}=(1 / 2) \min _{t \in[0, \tau]} y^{*}(t)$, there exists a $T_{1}>0$ such that

$$
y(t)>y^{*}(t)-\varepsilon_{0} \geq \frac{1}{2} \min _{t \in[0, \tau]} y^{*}(t) \triangleq m_{2},
$$

for all $t \geq T_{1}$. Together with Theorem 8 , we have that the predator species $y$ of system (4) is permanent. This completes the proof of Theorem 9 .

Remark 10. Set

$$
\theta=\int_{t}^{t+\tau}\left[\frac{(-1)^{k}+1}{2} a_{3}(s)+\frac{(-1)^{k+1}+1}{2} \widetilde{a}_{3}(s)\right] d s .
$$

In system (4), based on the assumptions and the actual biological meanings of the parameters $a_{3}(t), b_{3}(t)$ and $\widetilde{a}_{3}(t), \widetilde{b}_{3}(t)$, we can see that the conditions in Theorem 9 are easily satisfied. Additionally, the constant $\theta$ represents the minimal total growth rate of the predator species $y$. If $\theta>0$, which means that the predator species $y$ has another food resource. As a result, Theorem 9 implies that if $\theta>0$, then the predator species $y$ will be permanent regardless of whether the prey species $x$ exists or not. 
Next, on the permanence of the prey species $x$ of system (4), we have the following result.

Theorem 11. Suppose that assumptions $\left(H_{1}\right)-\left(H_{7}\right)$ and the following inequality

$$
\begin{aligned}
& \int_{t}^{t+\tau} \frac{(-1)^{k}+1}{2} a_{i}(s) \\
& +\frac{(-1)^{k+1}}{2}\left(\widetilde{a}_{i}(s)-c_{i}(s) y^{*}(s)\right) d s \\
& +\sum_{t \leq \tau_{k+1} \leq t+\tau} \ln h_{i k}>0
\end{aligned}
$$

for $(i=1,2, k \in N)$ hold. Then the prey species $x$ of system (4) is permanent.

Proof. Owing to condition (37), we have that there is a small enough constant $\varepsilon_{1}>0$, such that

$$
\begin{aligned}
& \int_{t}^{t+\tau} \frac{(-1)^{k}+1}{2}\left(a_{i}(s)-b_{i}(s) \varepsilon_{1}\right)+\frac{(-1)^{k+1}}{2}\left[\tilde{a}_{i}(s)\right. \\
& \left.-\widetilde{b}_{i}(s) \varepsilon_{1}-c_{i}(s)\left(y^{*}(s)+\varepsilon_{1}\right)\right] d s \\
& \quad+\sum_{t<\tau_{k+1} \leq(t+\tau)} \ln h_{i k}>0 .
\end{aligned}
$$

Consider the following auxiliary system:

$$
\begin{aligned}
& \dot{n}_{i}(t) \\
& \quad=n_{i}(t)\left[a_{i k}(t)-b_{i k}(t) n_{i}(t)-c_{i k}(t)\left(y^{*}(s)+\varepsilon_{1}\right)\right] \\
& \quad+d_{i j k}(t)\left[n_{j}\left(t-\tau_{i}\right)-n_{i}(t)\right], \quad t \in\left[\tau_{k}, \tau_{k+1}\right), \\
& \quad n_{i}\left(\tau_{k+1}\right)=h_{i k} n_{i}\left(\tau_{k+1}^{-}\right), \quad t=\tau_{k+1},
\end{aligned}
$$

where

$$
\begin{aligned}
& c_{1 k}(t)=\frac{(-1)^{k+1}+1}{2} c_{1}(t), \\
& c_{2 k}(t)=\frac{(-1)^{k+1}+1}{2} c_{2}(t) .
\end{aligned}
$$

By (38), we have

$$
\begin{aligned}
\int_{t}^{t+\tau} & \frac{(-1)^{k}+1}{2} a_{i}(s) \\
+ & \frac{(-1)^{k+1}}{2}\left[\widetilde{a}_{i}(s)-c_{i}(s)\left(y^{*}(s)+\varepsilon_{1}\right)\right] d s \\
+ & \sum_{t<\tau_{k+1} \leq(t+\tau)} \ln h_{i k}>0 .
\end{aligned}
$$

Then, from (41) and Lemma 5, we know system (39) has a unique globally attractive positive $\tau$-almost periodic solution $n_{\varepsilon_{1}}^{*}(t)=\left(n_{1 \varepsilon_{1}}^{*}(t), n_{2 \varepsilon_{1}}^{*}(t)\right)$.
And then, based on assumption $H_{8}$ and Lemma 5, for arbitrary constant $\gamma>0$ the following system

$$
\begin{aligned}
& \dot{u}(t)=\frac{(-1)^{k}+1}{2} u(t)\left[a_{3}(t)-b_{3}(t) u(t)\right] \\
& +\frac{(-1)^{k+1}+1}{2} u(t) \\
& \cdot\left[\tilde{a}_{3}(t)+\tilde{c}_{1}(t) \gamma+\tilde{c}_{2}(t) \gamma-\widetilde{b}_{3}(t) u(t)\right], \\
& u\left(\tau_{k+1}\right)=u\left(\tau_{k+1}^{-}\right), \quad t=\left[\tau_{k}, \tau_{k+1}\right),
\end{aligned}
$$

has a unique globally attractive positive $\tau$-almost periodic solution $u_{\gamma}^{*}(t)$. For above $\varepsilon_{1}>0$ and $\widehat{M}=\max \left\{M_{2}, m_{2}^{-1}\right\}>0$, there is a $t_{3}=t\left(\varepsilon_{1}, \widehat{M}\right)>0$, such that, for any $t_{0} \geq 0$ and $u_{0} \in\left[\widehat{M}^{-1}, \widehat{M}\right]$,

$$
\left|u_{\gamma}\left(t, t_{0}, u_{0}\right)-u_{\gamma}^{*}(t)\right|<\frac{\varepsilon_{1}}{2}
$$

for all $t \geq t_{0}+t_{3}$, where $u_{\gamma}\left(t, t_{0}, u_{0}\right)$ is the solution of system (42) with the initial condition $u_{\gamma}\left(t_{0}\right)=u_{0}$.

According to the theorem of the continuity of solutions with respect to parameters of the impulsive differential equations [17], there exist $\gamma_{0} \in\left(0, \varepsilon_{1}\right)$ and $\gamma_{0}<n_{\varepsilon_{1}}^{*}(t)-\gamma_{0}$ such that

$$
\left|u_{\gamma_{0}}^{*}(t)-y^{*}(t)\right|<\frac{\varepsilon_{1}}{2}
$$

for all $t \in[t, t+\tau]$. Also owing to the almost periodic property of $u_{\gamma_{0}}^{*}(t)$ and $y^{*}(t)$, we further have

$$
\left|u_{\gamma_{0}}^{*}(t)-y^{*}(t)\right|<\frac{\varepsilon_{1}}{2}
$$

for all $t \in R$.

Suppose $\left(x_{1}(t), x_{2}(t), y(t)\right)$ is arbitrary positive solution of system (4); from above inequalities (24), (31), and (35), we have

$$
\begin{aligned}
& x(t) \leq M_{1}, \\
& M^{-1} \leq y(t) \leq M
\end{aligned}
$$

for all $t \geq \widehat{T}=\max \left\{T_{1}, T_{2}\right\}$. If there exists a $t_{2} \geq \widehat{T}$, such that $x_{i}(t) \leq \gamma_{0}$ for all $t \geq t_{2}$, then from system (4), we can obtain

$$
\begin{aligned}
& \dot{y}(t) \leq \frac{(-1)^{k}+1}{2} y(t)\left[a_{3}(t)-b_{3}(t) y(t)\right] \\
& +\frac{(-1)^{k+1}+1}{2} y(t) \\
& \cdot\left[\tilde{a}_{3}(t)+\widetilde{c}_{1}(t) \gamma_{0}+\widetilde{c}_{2}(t) \gamma_{0}-\widetilde{b}_{3}(t) y(t)\right], \\
& y\left(\tau_{k+1}\right)=y\left(\tau_{k+1}^{-}\right), \quad t=\tau_{k+1},
\end{aligned}
$$


for all $t \geq t_{2}$. By comparison theorem of impulsive differential equations $[16,28,29]$, we also have $y(t) \leq u_{\gamma_{0}}(t)$ for all $t \geq t_{2}$, where $u_{\gamma_{0}}(t)$ is the solution of system (42) satisfying $\gamma=\gamma_{0}$ and with the initial value $u_{\gamma_{0}}\left(t_{2}\right)=y\left(t_{2}\right)$. For (43), let $t_{0}=t_{2}$ and $u\left(t_{0}\right)=y\left(t_{2}\right)$. From $M^{-1} \leq y(t) \leq M$, we have for $\gamma=\gamma_{0}$

$$
u_{\gamma_{0}}(t)=u_{\gamma_{0}}\left(t, t_{2}, y\left(t_{2}\right)\right)<u_{\gamma_{0}}^{*}(t)+\frac{1}{2} \varepsilon_{1},
$$

for all $t \geq t_{2}+t_{3}$. Then $y(t)<u_{\gamma_{0}}^{*}(t)+(1 / 2) \varepsilon_{1}$ for all $t \geq t_{2}+t_{3}$. Thus, by condition (45), we further get

$$
y(t)<y^{*}(t)+\varepsilon_{1}
$$

Taking into account system (4) again, from above inequality (49) and system (4), we have

$$
\begin{aligned}
& \quad \dot{x}_{i}(t) \\
& \quad \geq x_{i}(t)\left[a_{i k}(t)-b_{i k}(t) x_{i}(t)-c_{i k}(t)\left(y^{*}(t)+\varepsilon_{1}\right)\right] \\
& \quad+d_{i j k}(t)\left[x_{j}\left(t-\tau_{i}\right)-x_{i}(t)\right], \quad t \in\left[\tau_{k}, \tau_{k+1}\right), \\
& \quad x_{i}\left(\tau_{k+1}\right)=h_{i k} x_{i}\left(\tau_{k+1}^{-}\right), \quad t=\tau_{k+1},
\end{aligned}
$$

for all $t \geq t_{2}+t_{3}$. From Lemma 7, we also have $x_{i}(t) \geq$ $n_{i \varepsilon_{1}}(t)(i=1,2)$ for all $t \geq t_{2}+t_{3}$, where $n_{\varepsilon_{1}}(t)=$ $\left(n_{1 \varepsilon_{1}}(t), n_{2 \varepsilon_{1}}(t)\right)$ is the solution of system (39) satisfying the initial value $n_{i \varepsilon_{1}}\left(t_{2}+t_{3}\right)=x_{i}\left(t_{2}+t_{3}\right)$. Because of the globally attractivity of positive $\tau$-almost periodic solution $n_{\varepsilon_{1}}^{*}(t)$ of system (39), we further have that, for previous constant $\gamma_{0}$, there exists a $t_{4} \geq t_{2}+t_{3}$ such that

$$
n_{\varepsilon_{1}}(t) \geq n_{\varepsilon_{1}}^{*}(t)-\gamma_{0}>\gamma_{0}
$$

for all $t \geq t_{4}$. Hence, we have $x_{i}(t)>\gamma_{0}$ for all $t \geq t_{4}$, which leads to a contradiction.

Hence, there exists a constant $t_{5} \geq t_{2}$ such that $x_{i}\left(t_{5}\right)>\gamma_{0}$. We now prove that

$$
x_{i}(t) \geq \gamma_{0} \exp \left\{-\left[\left(M_{0}+\varphi_{0}\right) t_{3}+2 \xi_{i}\right]\right\} \quad \forall t \geq t_{5},
$$

where

$$
\begin{aligned}
\varphi_{0} & =\max _{t \in[0, \tau]}\left\{\mid a_{i k}(t)+b_{i k}(t) \gamma_{0}+c_{i k}(t)\left(y^{*}(t)+\varepsilon_{1}\right)\right. \\
& \left.+d_{i j k}(t) \mid\right\}, \\
M_{0} & =\min _{t \in[0, \tau]}\left\{\left|a_{i k}(t)-b_{i k}(t) M_{1}-c_{i k}(t) M-d_{i j k}(t)\right|\right\} .
\end{aligned}
$$

If inequality (52) is not true, then from $x_{i}\left(t_{5}\right)>\gamma_{0}>$ $\gamma_{0} \exp \left\{-\left[\left(M_{0}+\varphi_{0}\right) t_{3}+2 \xi_{i}\right]\right\}$, there are constants $t_{6}$ and $t_{7}$ satisfying $t_{7} \geq t_{6}>t_{5}$, such that $x_{i}\left(t_{7}\right)<\gamma_{0} \exp \left\{-\left[\left(M_{0}+\right.\right.\right.$ $\left.\left.\left.\varphi_{0}\right) t_{3}+2 \xi_{i}\right]\right\}, x_{i}\left(t_{6}^{-}\right) \geq \gamma_{0}, x_{i}\left(t_{6}\right) \leq \gamma_{0}$. There are two cases for $t_{7}$.

Case 1. $t_{6}=t_{7}$.

Case 2. $t_{6}<t_{7}$.
For Case 1, we see that $t_{6}$ is an impulsive time. Hence, there is an integer $k>0$ such that $t_{6}=t_{k}$. We have

$$
\begin{aligned}
\gamma_{0} \exp & \left\{-\left[\left(M_{0}+\varphi_{0}\right) t_{3}+2 \xi_{i}\right]\right\}>x_{i}\left(t_{6}\right)=h_{i k} x_{i}\left(t_{6}^{-}\right) \\
& \geq \gamma_{0} \exp \left(\ln h_{i k}\right) \geq \gamma_{0} \exp \left(-\xi_{i}\right) \\
& \geq \gamma_{0} \exp \left\{-\left[\left(M_{0}+\varphi_{0}\right) t_{3}+2 \xi_{i}\right]\right\}
\end{aligned}
$$

which leads to a contradiction.

For Case 2, we have $x_{i}(t)<\gamma_{0}$, for all $t \in\left(t_{6}, t_{7}\right]$. Assume $t_{7}-t_{6} \leq t_{3}$, then for any $t \in\left[t_{6}, t_{7}\right)$ we have

$$
\begin{aligned}
& \dot{x}_{i}(t) \\
& \quad \geq x_{i}(t)\left[a_{i k}(t)-b_{i k}(t) M_{1}-c_{i k}(t) M\right] \\
& \quad-d_{i j k}(t) x_{i}(t) \\
& \quad \geq x_{i}(t)\left[a_{i k}(t)-b_{i k}(t) M_{1}-c_{i k}(t) M-d_{i j k}(t)\right] \\
& \quad \geq x_{i}(t) M_{0}, \quad t \in\left[\tau_{k}, \tau_{k+1}\right), \\
& x_{i}\left(\tau_{k+1}\right)=h_{i k} x_{i}\left(\tau_{k+1}^{-}\right), \quad t=\tau_{k+1},
\end{aligned}
$$

where

$$
\begin{aligned}
& M_{0} \\
& \quad=\min _{t \in[0, \tau]}\left\{\left|a_{i k}(t)-b_{i k}(t) M_{1}-c_{i k}(t) M-d_{i j k}(t)\right|\right\} .
\end{aligned}
$$

Integrating (55) from $t_{6}$ to $t$, for any $t \in\left[t_{6}, t_{7}\right]$, we obtain

$$
\begin{aligned}
& x_{i}(t) \geq x_{i}\left(t_{6}\right) \\
& \cdot \exp \left\{\int_{t_{6}}^{t}\left[a_{i k}(s)-b_{i k}(s) M_{1}-c_{i k}(s) M-d_{i j k}(s)\right] d s\right. \\
& \left.\quad+\sum_{t_{6}<\tau_{k+1} \leq t} \ln h_{i k}\right\} \geq \gamma_{0} \exp \left[-\left(M_{0} t_{3}+\xi_{i}\right)\right] .
\end{aligned}
$$

Assume $t_{7}-t_{6}>t_{3}$; then when $t \in\left[t_{6}, t_{6}+t_{3}\right]$, according to above discussion we directly have

$$
x_{i}(t) \geq \gamma_{0} \exp \left[-\left(M_{0} t_{3}+\xi_{i}\right)\right] .
$$

Particularly,

$$
x_{i}\left(t_{6}+t_{3}\right) \geq \gamma_{0} \exp \left[-\left(M_{0} t_{3}+\xi_{i}\right)\right] .
$$

Since $x_{i}(t) \leq \gamma_{0}$ for all $t \in\left(t_{6}, t_{7}\right]$. Then we have

$$
\begin{aligned}
& \dot{y}(t) \leq \frac{(-1)^{k}+1}{2} y(t)\left[a_{3}(t)-b_{3}(t) y(t)\right] \\
& +\frac{(-1)^{k+1}+1}{2} y(t) \\
& \cdot\left[\tilde{a}_{3}(t)+\tilde{c}_{1}(t) \gamma_{0}+\widetilde{c}_{2}(t) \gamma_{0}-\tilde{b}_{3}(t) y(t)\right], \\
& \left.y\left(\tau_{k+1}\right)=y\left(\tau_{k+1}^{-}\right), \quad t=\tau_{k+1}, \tau_{k+1}\right),
\end{aligned}
$$


for $t \in\left(t_{6}, t_{7}\right]$. From comparison theorem of impulsive differential equations $[16,28,29]$, we also have $y(t) \leqslant u_{\gamma_{0}}(t)$ for all $t \in\left(t_{6}, t_{7}\right)$, where $u_{\gamma_{0}}(t)$ is the solution of system (42) with $\gamma=\gamma_{0}$ and satisfies $u_{\gamma_{0}}\left(t_{6}\right)=y\left(t_{6}\right)$. In (43), choose a $t_{0}=t_{6}$ and $u_{0}=y\left(t_{6}\right)$, since $M^{-1} \leq y\left(t_{6}\right) \leq M$, we have for $\gamma=\gamma_{0}$

$$
\begin{aligned}
& u_{\gamma_{0}}(t)=u_{\gamma_{0}}\left(t, t_{6}, y\left(t_{6}\right)\right) \leq u_{\gamma_{0}}^{*}(t)+\frac{1}{2} \varepsilon_{1} \\
& \forall t \geq t_{6}+t_{3} .
\end{aligned}
$$

Hence,

$$
y(t)<u_{\gamma_{0}}^{*}+\frac{\varepsilon_{1}}{2} \quad \forall t \geq t_{6}+t_{3} .
$$

Thus, from (45) we further have

$$
y(t)<y^{*}(t)+\varepsilon_{1} \quad \forall t \geq t_{6}+t_{3} .
$$

From (63), we have

$$
\begin{aligned}
& \dot{x}_{i}(t) \\
& \quad \geq x_{i}(t)\left[a_{i k}(t)-b_{i k}(t) \gamma_{0}-c_{i k}(t)\left(y^{*}(t)+\varepsilon_{1}\right)\right] \\
& \quad-d_{i j k}(t) x_{i}(t) \quad t \in\left[\tau_{k}, \tau_{k+1}\right), \\
& x_{i}\left(\tau_{k+1}\right)=h_{i k} x_{i}\left(\tau_{k+1}^{-}\right), \quad t=\tau_{k+1},
\end{aligned}
$$

For arbitrary $t \in\left[t_{6}+t_{3}, t_{7}\right)$. We choose an integer $p \geq 0$ such that $t_{7} \in\left[t_{6}+t_{3}+p \tau, t_{6}+t_{3}+(p+1) \tau\right)$; then integrating (64) from $t_{6}+t_{3}$ to $t_{7}$, we have

$$
\begin{aligned}
& \gamma_{0} \exp \left\{-\left[\left(M_{0}+\varphi_{0}\right) t_{3}+2 \xi_{i}\right]\right\}>x_{i}\left(t_{7}\right)=x_{i}\left(t_{6}+t_{3}\right) \\
& \cdot \exp \left\{\int _ { t _ { 6 } + t _ { 3 } } ^ { t _ { 7 } } \left[a_{i k}(s)-b_{i k}(s) \gamma_{0}\right.\right. \\
& \left.-c_{i k}(s)\left(y^{*}(s)+\varepsilon_{1}\right)-d_{i j k}(s)\right] d s \\
& \left.+\sum_{t_{6}+t_{3}<\tau_{k+1} \leq t_{7}} \ln h_{i k}\right\} \geq \gamma_{0} \exp \left[-\left(M_{0} t_{3}+\xi_{i}\right)\right] \\
& \cdot \exp \left\{( \int _ { t _ { 6 } + t _ { 3 } } ^ { t _ { 6 } + t _ { 3 } + p \omega } + \int _ { t _ { 6 } + t _ { 3 } + p \omega } ^ { t _ { 7 } } ) \left[a_{i k}(s)-b_{i k}(s) \gamma_{0}\right.\right. \\
& \left.-c_{i k}(s)\left(y^{*}(s)+\varepsilon_{1}\right)-d_{i j k}(s)\right] d s \\
& \left.+\sum_{t_{6}+t_{3}<\tau_{k+1} \leq t_{7}} \ln h_{i k}\right\} \geq \gamma_{0} \exp \left[-\left(M_{0} t_{3}+\xi_{i}\right)\right. \\
& \left.-\left(\varphi_{0} t_{3}+\xi_{i}\right)\right] \geq \gamma_{0} \exp \left\{-\left[\left(M_{0}+\varphi_{0}\right) t_{3}+2 \xi_{i}\right]\right\}
\end{aligned}
$$

which leads to a contradiction. According to the discussion above, we finally have

$$
\liminf _{t \rightarrow \infty} x_{i}(t) \geq m_{1} \triangleq \gamma_{0} \exp \left\{-\left[\left(M_{0}+\varphi_{0}\right) t_{3}+2 \xi_{i}\right]\right\} .
$$

Obviously, $m_{1}$ is independent of any positive solutions of system (4). This completes the proof of Theorem 11.
Remark 12. Set

$$
\begin{aligned}
\lambda & =\int_{t}^{t+\tau} \frac{(-1)^{k}+1}{2} a_{i}(s) \\
& +\frac{(-1)^{k+1}}{2}\left(\tilde{a}_{i}(s)-c_{i k}(s) y^{*}(t)\right) d s \\
& +\sum_{t \leq \tau_{k+1} \leq t+\tau} \ln h_{i k} .
\end{aligned}
$$

Since $y^{*}(t)$ is the globally asymptotically stable $\tau$ - periodic solution of the prey-free subsystem (14) of system (4), we obtain that the integral $\int_{t}^{t+\tau}\left(\left((-1)^{k}+1\right) / 2\right) a_{i}(s)+$ $\left((-1)^{k+1} / 2\right)\left(\widetilde{a}_{i}(s)-c_{i k}(s) y^{*}(t)\right) d s$ is the net total growth rate of species $x$ within a whole almost period, while $\sum_{t \leq \tau_{k+1} \leq t+\tau} \ln h_{i k}$ is the maximal total survivability of species $x$ within the process in which species $x$ disperses between patches. Therefore, $\lambda$ is the net total growth rate of species $x$ during a whole period of transference. Theorem 11 tells us that if $\lambda>0$, then species $x$ in system (4) is permanent.

\section{Numerical Simulation and Discussion}

In this paper, an almost periodic predator-prey model with intermittent prey dispersals and intermittent predation has been investigated. By using the methods of analysis and comparison theorems of the impulsive differential equations, we obtain sufficient conditions of the boundedness and permanence for this system.

To illustrate our results for system (4), we give some numerical simulations by using the following values of parameters in Table 1.

Due to system (4) being an almost periodic system, we will show the numerical simulation on the following eight intervals in Table 2.

Therefore, we can consider almost periodic system as periodic approximately and the $\tau$ - period is 10 .

First, for system (12), when we take each parameter as in Table 1, we have

$$
\begin{aligned}
& \int_{0}^{10} a_{1}(s) d s+\sum_{0<\tau_{k+1} \leq 10} \ln d_{1} \approx 4.3918>0, \\
& \int_{0}^{10} \tilde{a}_{1}(s) d s+\sum_{0<\tau_{k+1} \leq 10} \ln D_{1} \approx 5.4615>0, \\
& \int_{0}^{10} a_{2}(s) d s+\sum_{0<\tau_{k+1} \leq 10} \ln d_{2} \approx 5.0316>0, \\
& \int_{0}^{10} \tilde{a}_{2}(s) d s+\sum_{0<\tau_{k+1} \leq 10} \ln D_{2} \approx 5.6074>0 .
\end{aligned}
$$

We can see that all the assumptions $\left(H_{1}\right)-\left(H_{7}\right)$ of Lemma 5 hold. Therefore, system (12) has a unique positive globally attractive almost periodic solution $x^{*}(t)=\left(x_{1}^{*}(t), x_{2}^{*}(t)\right)$. Numerical simulations of these results can be observed in Figures 1(a) and 1(b). From numerical simulations, we obtain 
TABLE 1: Parameter values used in the simulations of system (4).

\begin{tabular}{lc}
\hline Parameter & Value \\
\hline$a_{1}(t)$ & $0.5+0.2 \sin (1.2 \pi t)+0.2 \sin (1.5 \pi t)$ \\
$\widetilde{a}_{1}(t)$ & $0.6+0.14 \sin (1.2 \pi t)+0.14 \sin (1.5 \pi t)$ \\
$a_{2}(t)$ & $0.55+0.1 \sin (1.2 \pi t)+0.1 \sin (1.5 \pi t)$ \\
$\tilde{a}_{2}(t)$ & $0.6+0.09 \sin (1.2 \pi t)+0.09 \sin (1.5 \pi t)$ \\
$a_{3}(t)$ & $0.5+0.3 \sin (1.2 \pi t)+0.2 \sin (1.5 \pi t)$ \\
$\widetilde{a}_{3}(t)$ & $0.1+0.12 \sin (1.2 \pi t)+0.12 \sin (1.5 \pi t)$ \\
$b_{1}(t)$ & $0.25+0.1 \cos (1.2 \pi t)+0.1 \cos (1.5 \pi t)$ \\
$\widetilde{b}_{1}(t)$ & $0.4+0.04 \cos (1.2 \pi t)+0.04 \cos (1.5 \pi t)$ \\
$b_{2}(t)$ & $0.3+0.04 \cos (1.2 \pi t)+0.04 \cos (1.5 \pi t)$ \\
$\widetilde{b}_{2}(t)$ & $0.4+0.05 \cos (1.2 \pi t)+0.05 \cos (1.5 \pi t)$ \\
$b_{3}(t)$ & $0.2+0.1 \cos (1.2 \pi t)+0.2 \cos (1.5 \pi t)$ \\
$\widetilde{b}_{3}(t)$ & $0.5+0.10 \cos (1.2 \pi t)+0.10 \cos (1.5 \pi t)$ \\
$c_{1}(t)$ & $0.2+0.2 \sin (1.2 \pi t)+0.2 \sin (1.5 \pi t)$ \\
$\widetilde{c}_{1}(t)$ & $0.10+0.1 \sin (1.2 \pi t)+0.1 \sin (1.5 \pi t)$ \\
$c_{2}(t)$ & $0.15+0.2 \sin (1.2 \pi t)+0.2 \sin (1.5 \pi t)$ \\
$\widetilde{c}_{2}(t)$ & $0.15+0.2 \sin (1.2 \pi t)+0.2 \sin (1.5 \pi t)$ \\
$D_{12}(t)$ & $0.5+0.05 \cos (1.2 \pi t)+0.05 \cos (1.5 \pi t)$ \\
$D_{21}(t)$ & $0.5+0.05 \cos (1.2 \pi t)+0.05 \cos (1.5 \pi t)$ \\
$d_{1}$ & 0.5 \\
$d_{2}$ & 0.6 \\
$D_{1}$ & 0.55 \\
$D_{2}$ & 0.65 \\
$\tau_{1}$ & 1.3 \\
$\tau_{2}$ & 1.6 \\
\hline &
\end{tabular}

the minimum values of $\left(x_{1}(t), x_{2}(t)\right)$, which are $(0.6988$, 0.8355).

Then, for system (14), when we also take the above parameters $a_{3 k}(t), b_{3 k}(t)$, we have

$$
\begin{gathered}
\int_{0}^{10} a_{3}(s) d s+\sum_{0<\tau_{k+1} \leq 10} \ln 1 \approx 5.0509>0, \\
\int_{0}^{10} \tilde{a}_{3}(s) d s+\sum_{0<\tau_{k+1} \leq 10} \ln 1 \approx 1.0509>0 .
\end{gathered}
$$

We can easily demonstrate that the assumptions $\left(H_{1}\right)-\left(H_{2}\right)$ and $\left(H_{7}\right)-\left(H_{8}\right)$ of Lemma 6 and Theorem 9 hold. Obviously, system (14) has a unique almost periodic solution $y^{*}(t)$, and the predator species $y$ of system (4) always has the minimum value of 0.3528 and the maximum value of 1.9017 when taking different initial values; that is, it is permanent. All of these results can be observed in Figure 2(a). However, for system (4), when we keep the other parameters unchanged and only just adjust the value of $\widetilde{a}_{3}(t)=-0.65+0.12 \sin (1.2 \pi t)+$ $0.12 \sin (1.5 \pi t)$, then we have

$$
\int_{0}^{10} \tilde{a}_{3}(s) d s+\sum_{0<\tau_{k+1} \leq 10} \ln 1 \approx-6.4491<0 .
$$

This is contrary to the assumptions $\left(H_{1}\right)-\left(H_{2}\right)$ and $\left(H_{7}\right)$ $\left(H_{8}\right)$ of Theorem 9, that is, in the whole period, if $\theta<0$ without considering the prey species $x$, then the predator species $y$ of system (4) will be extinct (see Figure 2(b)). Nevertheless, if we take the prey species $x$ into account, using the above parameters $\widetilde{c}_{1}(t), \widetilde{c}_{2}(t)$ and the minimum values of $\left(x_{1}(t), x_{2}(t)\right)$ with $0.6988,0.8355$ to make

$$
\begin{aligned}
& \int_{0}^{5} a_{3}(s) d s \\
& \quad+\int_{5}^{10}\left[\widetilde{a}_{3}(s)+0.6988 \times \widetilde{c}_{1}(s)+0.8355 \times \widetilde{c}_{2}(s)\right] d s \\
& \quad+\sum_{0<\tau_{k+1} \leq 10} \ln 1 \approx 0.6275>0,
\end{aligned}
$$

then we find an interesting phenomenon where the predator species $y$ of system (4) goes from extinct to permanent (see Figure 3(a)). It turns out that if the predator species $y$ in the time intervals $\left[\tau_{2 k+1}, \tau_{2 k+2}\right)$ has no other food resource but can only rely on the prey species $x$, then the more the predation behavior happens, the more likely the predator species will be permanent.

Moreover, to illustrate the difference between continuous and intermittent predation of the predator species $y$, we consider the following system [25]:

$$
\begin{aligned}
& \dot{x}_{1}(t) \\
& =x_{1}(t)\left[a_{1}(t)-b_{1}(t) x_{1}(t)-c_{1}(t) y(t)\right] \\
& \quad+D_{12}(t)\left[x_{2}(t)-x_{1}(t)\right], \\
& \dot{x}_{2}(t) \\
& =x_{2}(t)\left[a_{2}(t)-b_{2}(t) x_{2}(t)-c_{2}(t) y(t)\right] \\
& \quad+D_{21}(t)\left[x_{1}(t)-x_{2}(t)\right],
\end{aligned}
$$

$\dot{y}(t)$

$$
=y(t)\left[a_{3}(t)-b_{3}(t)+\widetilde{c}_{1}(t) x_{1}(t)+\widetilde{c}_{2}(t) x_{2}(t)\right] .
$$

We keep the other parameters unchanged including the intrinsic growth rates of the species $x$, the interspecies competition rates of all species, the survival rates of the species $x$, and the dispersal rates of the species $x$ in system (72). We take another set of parameters in Table 3, and we let $\tau_{i}=0, d_{i}=D_{i}=1(i=1,2)$ for the purpose of contrast.

By simple calculation, we have

$$
\begin{aligned}
& \int_{0}^{10}\left[a_{3}(s)+0.6988 \times \widetilde{c}_{1}(s)+0.8355 \times \widetilde{c}_{2}(s)\right] d s \\
& +\sum_{0<\tau_{k+1} \leq 10} \ln 1 \approx 5.7530>0 \\
& \int_{0}^{10}\left[a_{1}(s)-1.9017 \times c_{1}(s)\right] d s+\sum_{0<\tau_{k+1} \leq 10} \ln 1 \\
& \quad \approx-0.7009<0, \\
& \int_{0}^{10}\left[a_{2}(s)-1.9017 \times c_{2}(s)\right] d s+\sum_{0<\tau_{k+1} \leq 10} \ln 1 \\
& \approx-0.2434<0 .
\end{aligned}
$$


TABLE 2: Parameter values used in the simulations of system (4).

\begin{tabular}{lcccc}
\hline Number & Interval & $\tau_{k+1}-\tau_{k}$ & Interval length & Process \\
\hline 1 & {$[0,5)$} & $\tau_{1}-\tau_{0}$ & 5 & 1 \\
2 & {$[5,9)$} & $\tau_{2}-\tau_{1}$ & 4 & 2 \\
3 & {$[14,20)$} & $\tau_{3}-\tau_{2}$ & 6 & 1 \\
4 & {$[20,25)$} & $\tau_{4}-\tau_{3}$ & 5 & 2 \\
5 & {$[25,28)$} & $\tau_{5}-\tau_{4}$ & 3 & 1 \\
6 & {$[28,35)$} & $\tau_{6}-\tau_{5}$ & 7 & 2 \\
7 & {$[40,46)$} & $\tau_{7}-\tau_{6}$ & 6 & \\
8 & {$[46,50)$} & $\tau_{8}-\tau_{7}$ & 4 & 2 \\
\hline
\end{tabular}

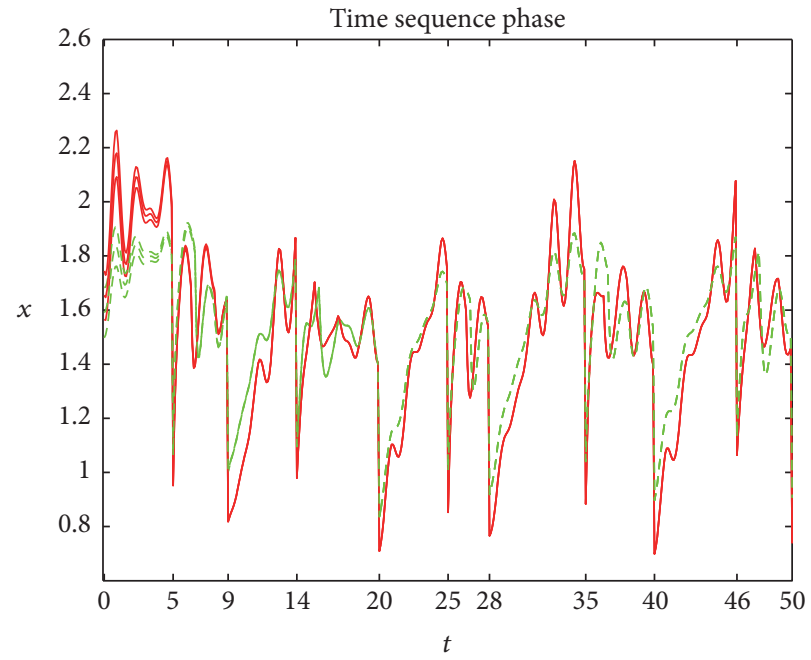

$\begin{array}{rr}- & x 1 \\ --- & x 2\end{array}$

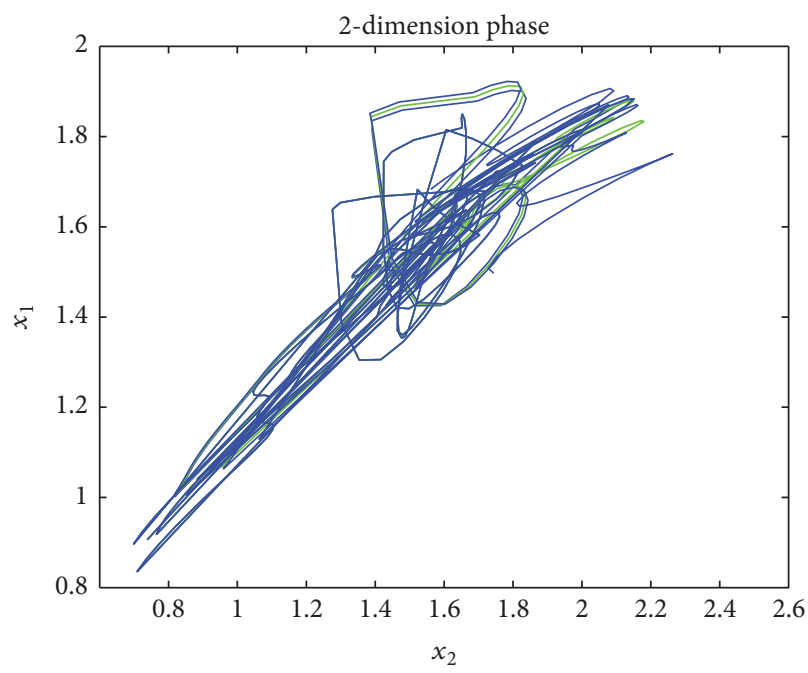

(a)

(b)

FIGURE 1: (a) The globally stable positive almost periodic solution of the species $x$ of system (12). (b) The portrait of the globally stable positive almost periodic solution of the species $x$ of system $(12)$. Here, we take the initial values $\phi_{i}(s)=\left(\phi_{1}(s), \phi_{2}(s)\right)=(1.5,1.8),(1.6,1.7),(1.7,1.6)$ for all $s \in[-5,0]$.

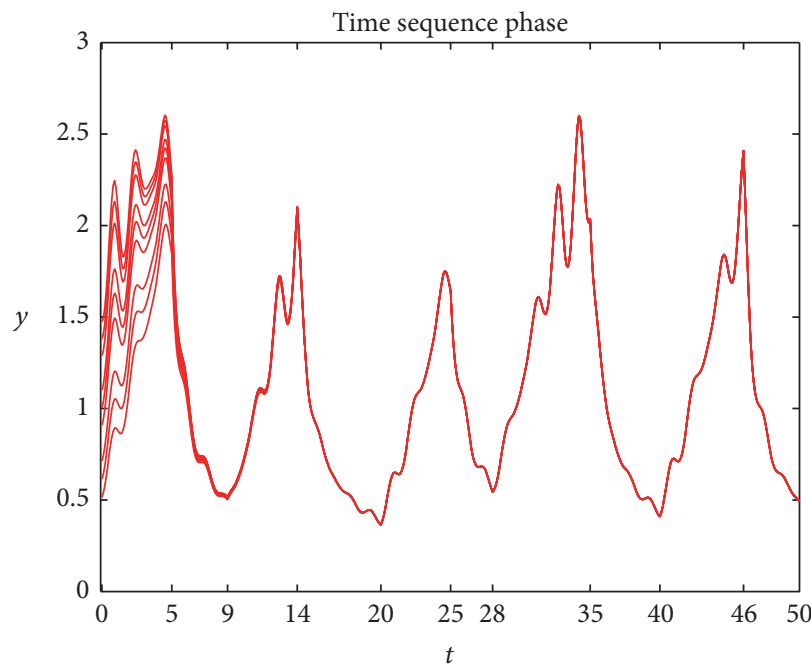

(a)

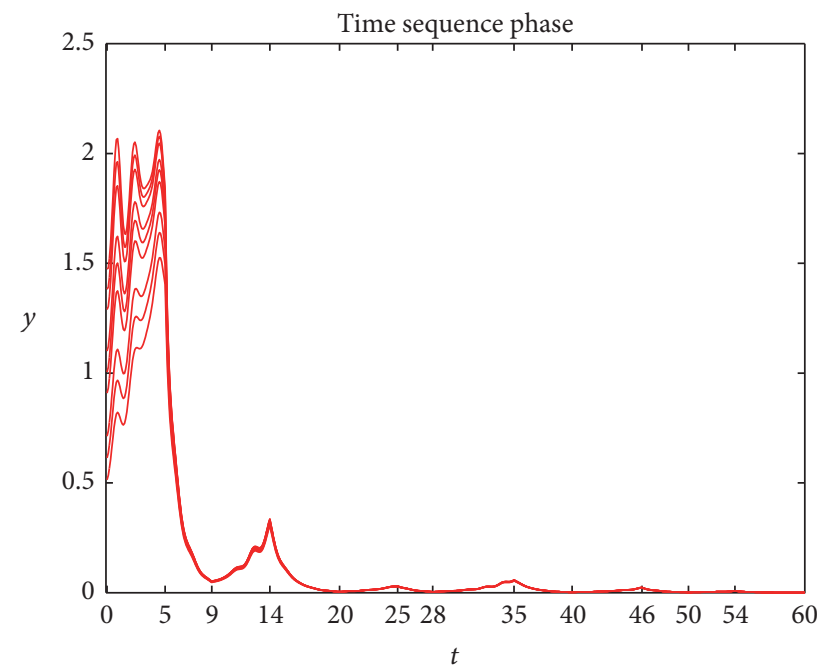

(b)

FIgURE 2: (a) The globally stable positive almost periodic solution of the species $y$ of system (14), and we take the initial condition $\phi_{i}(s)=$ $(1.2,0.8,0.4)$ for all $s \in[-5,0]$. (b) Extinction of the species $y$ of system $(14)$, and we take the initial values $\phi_{i}(s)=(0.8,0.4)$ for all $s \in[-5,0]$. 

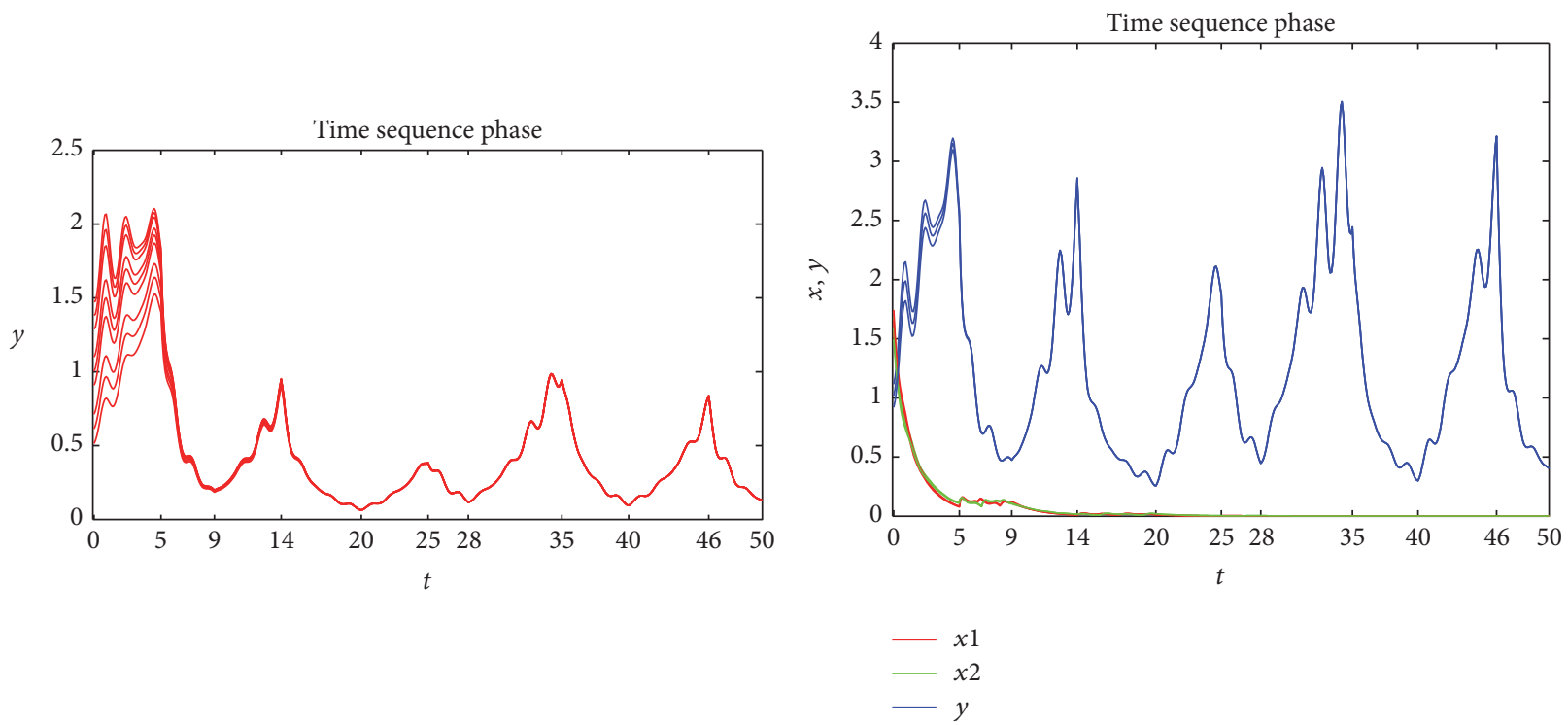

(a)

(b)

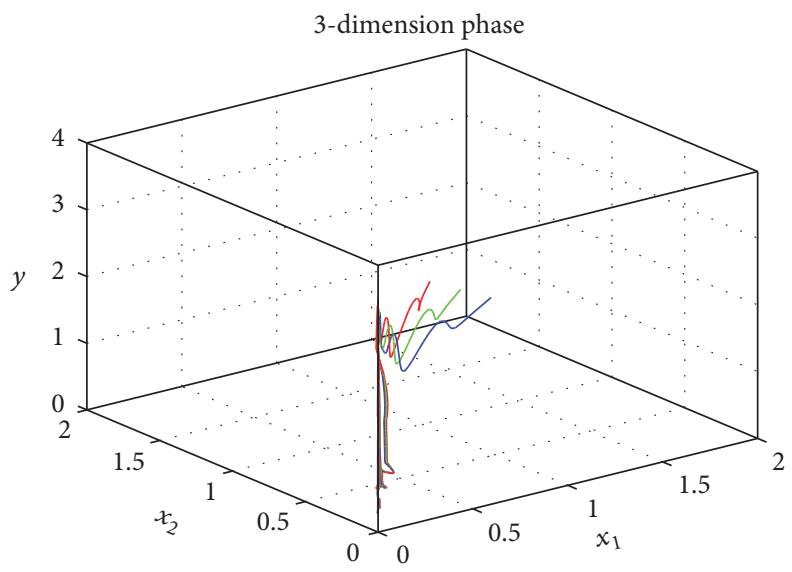

(c)

Figure 3: (a) The globally stable positive almost periodic solution of the species $y$ of system (14), and the predator species $y$ is permanent just relying on the predation; here, we take the initial condition $\phi_{i}(s)=(1.2,0.8,0.4)$ for all $s \in[-5,0]$. (b) The time series of almost periodic solution of the species $x, y$ of system (72). (c) The 3-dimension time series of almost periodic solution of the species $x, y$ of system (72).

TABLE 3: Parameter values used in the simulations of system (4).

\begin{tabular}{lc}
\hline Parameter & Value \\
\hline$a_{3}(t)$ & $0.1+0.3 \sin (1.2 \pi t)+0.2 \sin (1.5 \pi t)$ \\
$\tilde{a}_{3}(t)$ & $-0.6+0.12 \sin (1.2 \pi t)+0.12 \sin (1.5 \pi t)$ \\
$c_{1}(t)$ & $0.3+0.1 \sin (1.2 \pi t)+0.1 \sin (1.5 \pi t)$ \\
$\tilde{c}_{1}(t)$ & $0.3+0.14 \sin (1.2 \pi t)+0.1 \sin (1.5 \pi t)$ \\
$c_{2}(t)$ & $0.3+0.1 \sin (1.2 \pi t)+0.1 \sin (1.5 \pi t)$ \\
$\tilde{c}_{2}(t)$ & $0.3+0.14 \sin (1.2 \pi t)+0.1 \sin (1.5 \pi t)$ \\
\hline
\end{tabular}

Then, we find that continuous predation makes the predator permanent and the prey extinct (see Figures 3(b) and 3(c)). However, if we keep all others parameters unchanged and just change system (72) to system (4), that is, changing the model from continuous predation to intermittent predation, we have

$$
\begin{aligned}
& \int_{0}^{5} a_{3}(s) d s \\
& \quad+\int_{5}^{10}\left[\tilde{a}_{3}(s)+0.6988 \times \tilde{c}_{1}(s)+0.8355 \times \widetilde{c}_{2}(s)\right] d s
\end{aligned}
$$




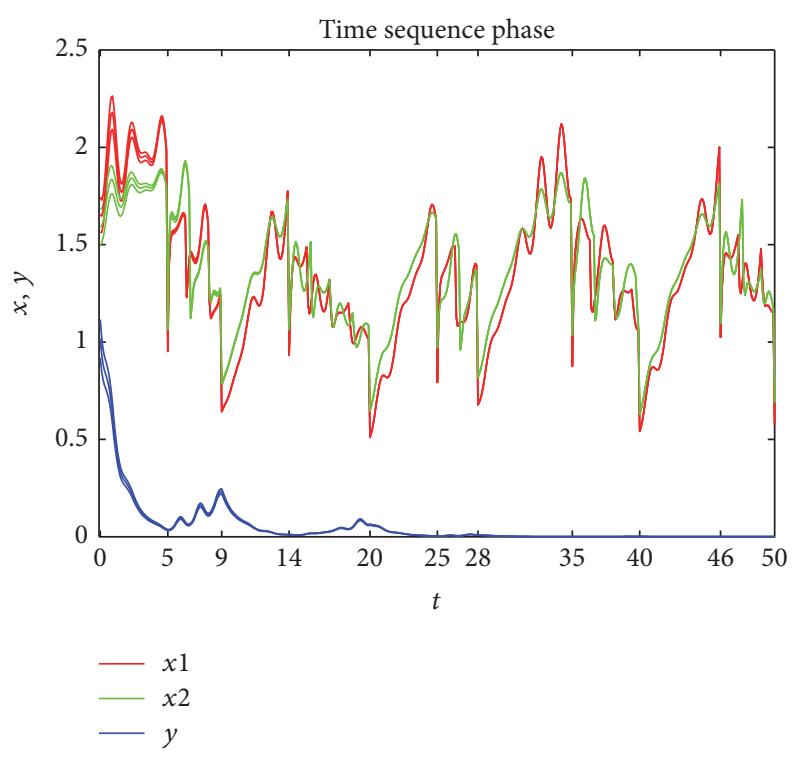

(a)

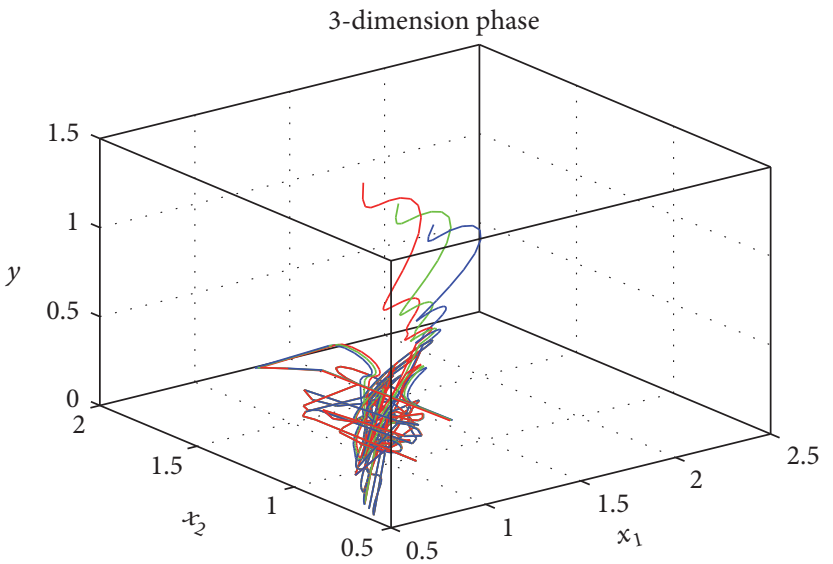

(b)

FIGURE 4: (a) The time series of almost periodic solution of the species $x$ of the predator-prey model with continuous predation. (b) The time series of almost periodic solution of the species $x$ of the predator-prey model with intermittent predation. Here, we take the initial condition $\phi_{i}(s)=\left(\phi_{1}(s), \phi_{2}(s)\right)=(1.5,1.8),(1.6,1.7),(1.7,1.6)$ for all $s \in[-5,0]$.

$$
\begin{aligned}
& +\sum_{0<\tau_{k+1} \leq 10} \ln 1 \approx-0.0981<0, \\
& \int_{0}^{5} a_{1}(s) d s+\int_{5}^{10}\left[\widetilde{a}_{1}(s)-1.9017 \times c_{1}(s)\right] d s \\
& +\sum_{0<\tau_{k+1} \leq 10} \ln 1 \approx 2.6762>0, \\
& \int_{0}^{5} a_{2}(s) d s+\int_{5}^{10}\left[\widetilde{a}_{1}(s)-1.9017 \times c_{2}(s)\right] d s \\
& +\sum_{0<\tau_{k+1} \leq 10} \ln 1 \approx 2.8974>0 .
\end{aligned}
$$

Then, there are some interesting phenomena that the predator goes from permanent to extinct, while the opposite occurs for the prey. All of these results can be observed (Figures 4(a) and $4(\mathrm{~b}))$ by numerical simulations. By the above analysis, we can conclude that if the predator continues preying on prey and the predation intensity is higher than the intrinsic growth of the prey, then the prey will be extinct, while the predator will be permanent. We also obtain that if the predation behavior is intermittent and the predation intensity is small, then the predator will be extinct, while the prey will be permanent. So, for an intermittent predator-prey model, both the intermittent predation and the intrinsic growth rates of the prey and predator species can greatly impact the permanence or extinction of the system.

Finally, for system (4), to test our main results, we use the above parameters in Table 1. We can simply calculate that the assumptions $\left(H_{1}\right)-\left(H_{7}\right)$ in Theorem 11 hold, and for (37), we let $y^{*}(t)=1.9017$ then

$$
\begin{aligned}
& \int_{0}^{10} a_{1}(s) d s+\sum_{0<\tau_{k+1} \leq 10} \ln d_{1} \approx 4.3918>0, \\
& \int_{0}^{10}\left[\widetilde{a}_{1}(s)-1.9017 \times \widetilde{c}_{1}(s)\right] d s+\sum_{0<\tau_{k+1} \leq 10} \ln D_{1} \\
& \approx 1.4967>0, \\
& \int_{0}^{10} a_{2}(s) d s+\sum_{0<\tau_{k+1} \leq 10} \ln d_{2} \approx 5.0316>0, \\
& \int_{0}^{10}\left[\widetilde{a}_{2}(s)-1.9017 \times \widetilde{c}_{1}(s)\right] d s+\sum_{0<\tau_{k+1} \leq 10} \ln D_{2} \\
& \approx 2.5934>0 .
\end{aligned}
$$

From numerical simulations, we obtain that the minimum value of $x$ of system (3) is always 0.8085 when using different initial values; that is, the prey $x$ is permanent (see Figures 5(a) and 5(b)).

\section{Conflicts of Interest}

All the authors declare that there are no conflicts of interest regarding the publication of this paper. 


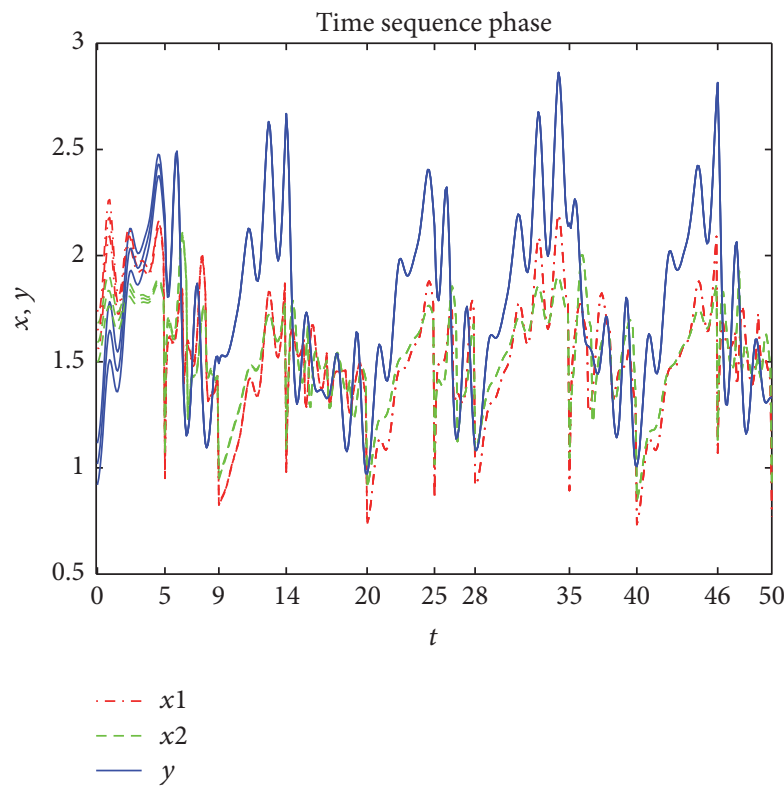

(a)

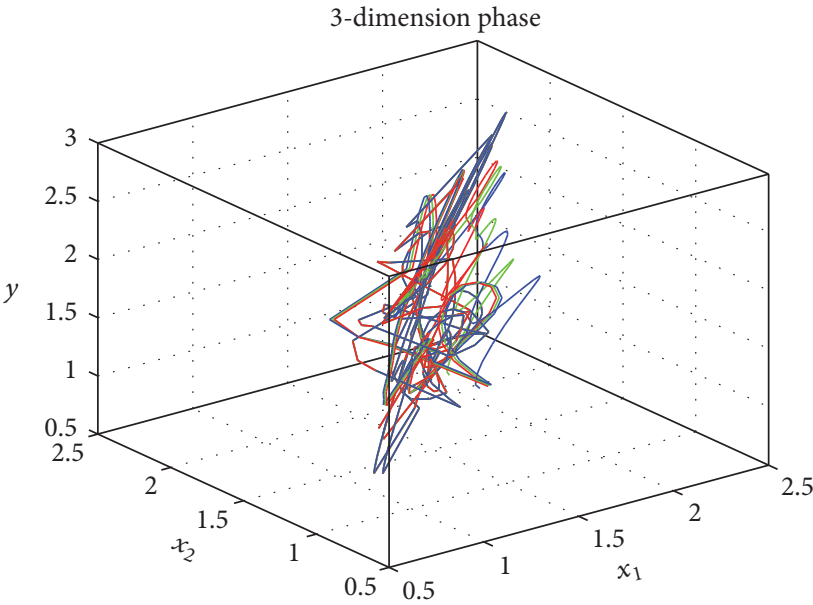

(b)

Figure 5: (a) The time series of the globally stable positive almost periodic solution of the species $x$. (b) The portrait of the globally stable positive almost periodic solution of the species $x$, and we take the initial condition $\phi_{i}(s)=\left(\phi_{1}(s), \phi_{2}(s)\right)=(1.5,1.8),(1.6,1.7),(1.7,1.6)$ for all $s \in[-5,0]$.

\section{Acknowledgments}

This work was supported by the National Natural Science Foundation of China (11361059, 11702237), the Key Project of Laboratory of Xinjiang Province of China (2016D03022), the NSF of Jiangsu Province of China (BK20130799), and the Doctoral Scientific Research Foundation of Xinjiang University (no. BS160204).

\section{References}

[1] L. Zhang and Z. Teng, "Permanence in a periodic predator-prey system with prey dispersal and predator density-independent," Journal of Biological Systems, vol. 14, no. 4, pp. 491-507, 2006.

[2] S. K. Sasmal and D. Ghosh, "Effect of dispersal in two-patch prey-predator system with positive density dependence growth of preys," BioSystems, vol. 151, pp. 8-20, 2017.

[3] P. Gramlich, S. J. Plitzko, L. Rudolf, B. Drossel, and T. Gross, "The influence of dispersal on a predator-prey system with two habitats," Journal of Theoretical Biology, vol. 398, pp. 150-161, 2016.

[4] X. Song and L. Chen, "Harmless delays and global attractivity for nonautonomous predator-prey system with dispersion," Computers and Mathematics with Applications. An International Journal, vol. 39, no. 5-6, pp. 33-42, 2000.

[5] J. A. Sherratt, "Invasion generates periodic traveling waves (wavetrains) in predator-prey models with nonlocal dispersal," SIAM Journal on Applied Mathematics, vol. 76, no. 1, pp. 293313, 2016.

[6] Z. Teng and L. Chen, "Permanence and extinction of periodic predator-prey systems in a patchy environment with delay," Nonlinear Analysis: Real World Applications, vol. 4, no. 2, pp. 335-364, 2003.
[7] S. Pan, "Minimal wave speeds of delayed dispersal predatorprey systems with stage structure," Electronic Journal of Differential Equations, Paper No. 121, 16 pages, 2016.

[8] Z. Teng, "Nonautonomous Lotka-Volterra systems with delays," Journal of Differential Equations, vol. 179, no. 2, pp. 538-561, 2002.

[9] L.-L. Wang and W.-T. Li, "Existence and global stability of positive periodic solutions of a predator-prey system with delays," Applied Mathematics and Computation, vol. 146, no. 1, pp. 167-185, 2003.

[10] K. Cheng, T.-H. Yang, and J.-W. Yu, "Dynamics of the predatorprey models on the two-patch fragmented habitat with dispersal," Tamkang Journal of Mathematics, vol. 47, no. 1, pp. 127-141, 2016.

[11] K. Wang, "Persistence for nonautonomous predator-prey systems with infinite delay," Acta Mathematica Sinica, vol. 40, no. 3, pp. 321-332, 1997.

[12] R. Xu, M. A. Chaplain, and F. A. Davidson, "Periodic solutions for a delayed predator-prey model of prey dispersal in twopatch environments," Nonlinear Analysis: Real World Applications, vol. 5, no. 1, pp. 183-206, 2004.

[13] R. Xu, M. A. Chaplain, and F. A. Davidson, "Periodic solution of a Lotka-Volterra predator-prey model with dispersion and time delays," Applied Mathematics and Computation, vol. 148, no. 2, pp. 537-560, 2004.

[14] H.-L. Li, L. Zhang, Z.-D. Teng, and Y.-L. Jiang, "A delayed predator-prey system with impulsive diffusion between two patches," International Journal of Biomathematics, vol. 10, no. 1, 1750010, 20 pages, 2017.

[15] L. X. Zhao and J. J. Chen, "Analysis on migratory routine of Larimichthy polyactis," Journal of Fishery Sciences of China, vol. 16, pp. 931-940, 2009. 
[16] D. Bainov and P. Simeonov, Impulsive Differential Equations: Periodic Solutions and Applications, Longman Scientific and Technical, New York, NY, USA, 1993.

[17] S. Ahmad and I. M. Stamova, "Asymptopic stability of a Ndimesensional impulsive competitive system," Nonlinear Analysis: Real World Applications, vol. 8, no. 2, pp. 654-663, 2007.

[18] J. Hou, Z. Teng, and S. Gao, "Permanence and global stability for nonautonomous $\mathrm{N}$-species Lotka-Valterra competitive system with impulses," Nonlinear Analysis: Real World Applications, vol. 11, no. 3, pp. 1882-1896, 2010.

[19] Z. Jin, M. Zhien, and H. Maoan, "The existence of periodic solutions of the N-species Lotka-Volterra competitive systems with impulsive," Chaos Soliton Fractals, vol. 22, no. 1, pp. 181$188,2004$.

[20] L. Zhang, Z. Teng, and H. Jiang, "Permanence for general nonautonomous impulsive population systems of functional differential equations and its applications," Acta Applicandae Mathematicae, vol. 110, no. 3, pp. 1169-1197, 2010.

[21] L. Zhang, Z. Teng, D. L. DeAngelis, and S. Ruan, "Single species models with logistic growth and dissymmetric impulse dispersal," Mathematical Biosciences, vol. 241, no. 2, pp. 188-197, 2013.

[22] L. Zhang, Z. Teng, and Z. Liu, "Survival analysis for a periodic predator-prey model with prey impulsively unilateral diffusion in two patches," Applied Mathematical Modelling, vol. 35, no. 9, pp. 4243-4256, 2011.

[23] Y. Shao, "Analysis of a delayed predator-prey system with impulsive diffusion between two patches," Mathematical and Computer Modelling, vol. 52, no. 1-2, pp. 120-127, 2010.

[24] L. Zhang and Z. Teng, "The dynamical behavior of a predatorprey system with Gompertz growth function and impulsive dispersal of prey between two patches," Mathematical Methods in the Applied Sciences, vol. 39, no. 13, pp. 3623-3639, 2016.

[25] I. Newton, The migration ecology of birds, Academic Press, 2010.

[26] L. Zhang, G. Xu, and Z. Teng, "Intermittent dispersal population model with almost period parameters and dispersal delays," Discrete and Continuous Dynamical Systems - Series B, vol. 21, no. 6, pp. 2011-2037, 2016.

[27] K. M. Kostyal, Great Migrates, Dolphin Books Press, Beijing, China, 2011.

[28] X. Fu, B. Yan, and Y. Liu, Introduction to Impulsive Differential Systems, Science Press, Beijing, China, 2005.

[29] V. Lakshmikantham, D. Bainov, and P. Simeonov, Theorem of Impulsive Differential Equations, World Science, Singapore, 1989.

[30] C. Y. He, Almost Periodic Differential Equations, Higher Eduction Press, Beijing, China, 1992.

[31] Y. Kuang, Delay Differential Equations with Applications in Population Dynamics, Academic Press, New York, NY, USA, 1993. 


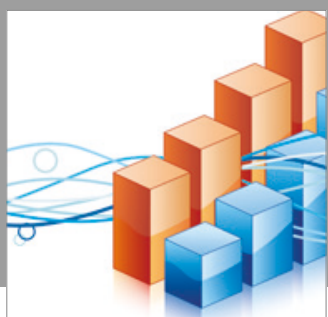

Advances in

Operations Research

vatersals

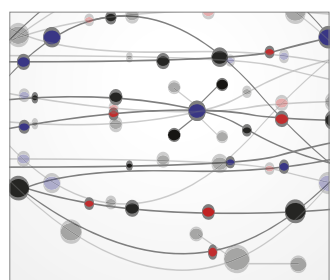

\section{The Scientific} World Journal
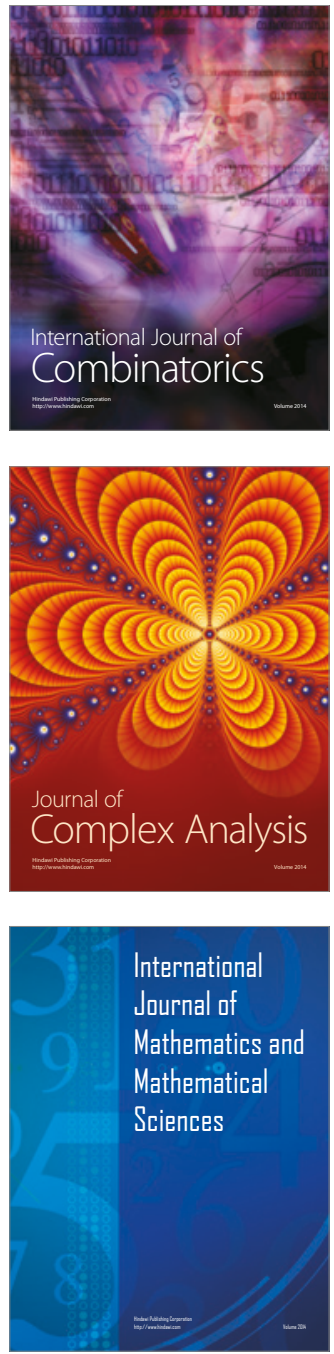
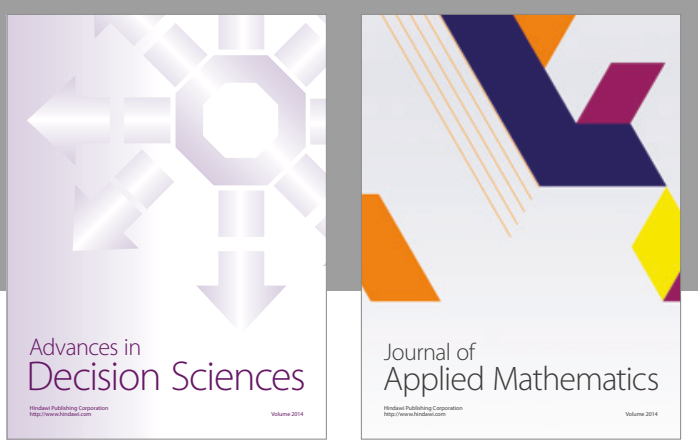

Algebra

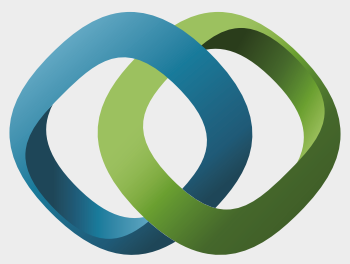

\section{Hindawi}

Submit your manuscripts at

https://www.hindawi.com
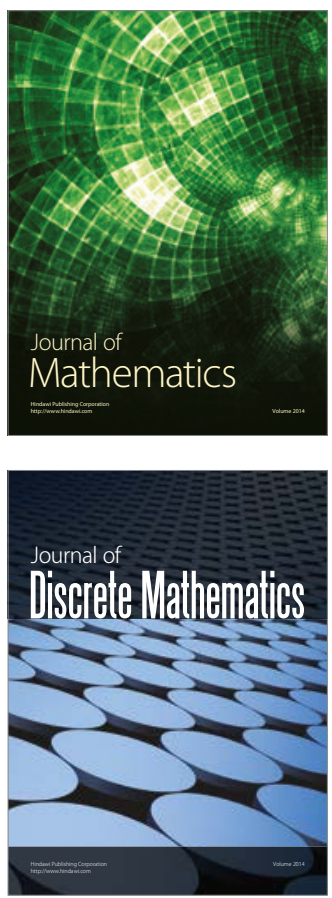

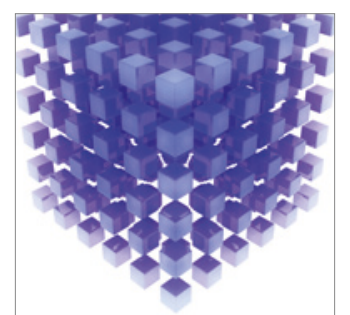

Mathematical Problems in Engineering
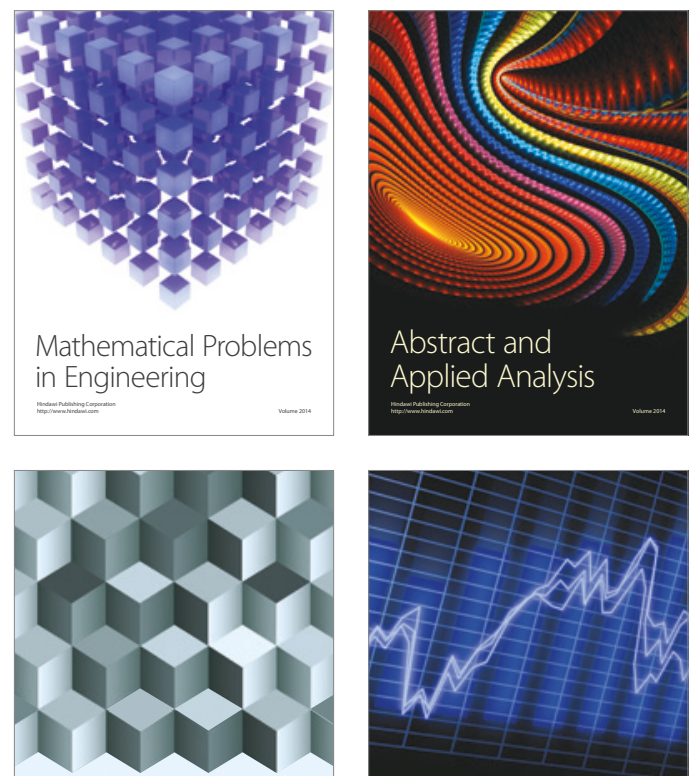

Journal of

Function Spaces

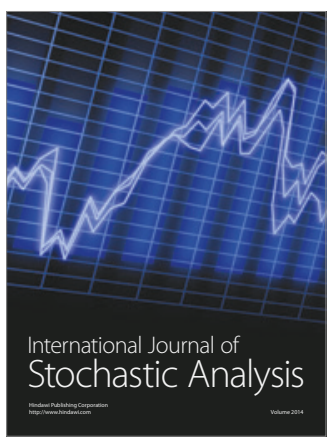

Probability and Statistics
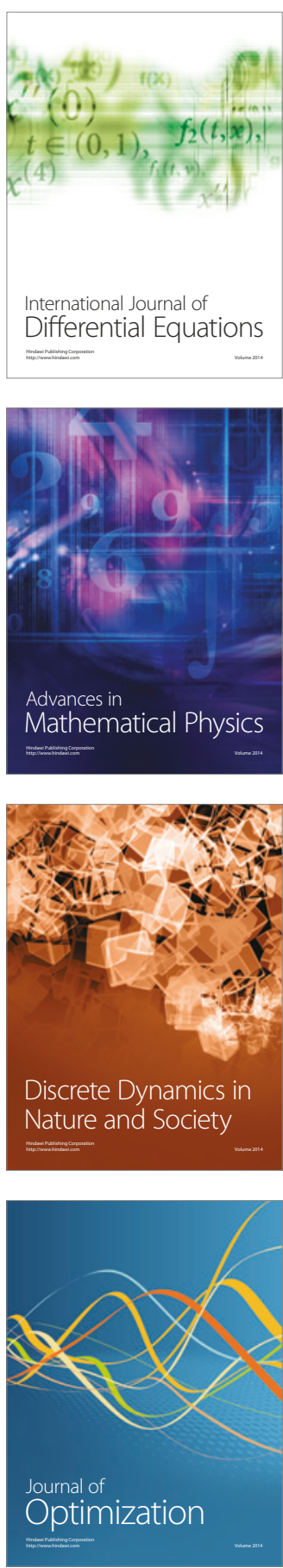